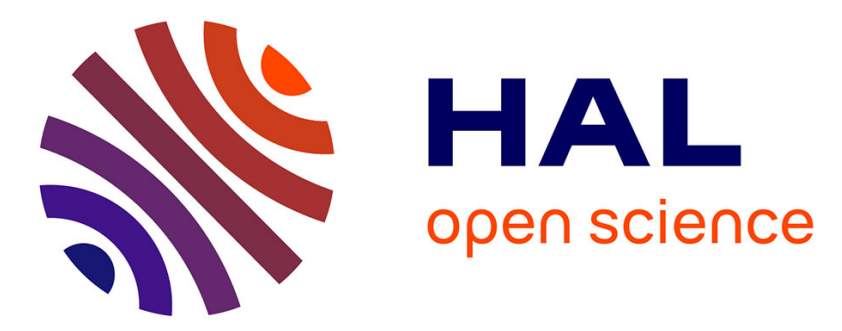

\title{
Space Physics Statistics of electric fields' amplitudes in Langmuir turbulence: A numerical simulation study
}

\author{
A. Voshchepynets, A Volokitin, Vladimir Krasnoselskikh, C Krafft
}

\section{To cite this version:}

A. Voshchepynets, A Volokitin, Vladimir Krasnoselskikh, C Krafft. Space Physics Statistics of electric fields' amplitudes in Langmuir turbulence: A numerical simulation study. Journal of Geophysical Research Space Physics, 2017, 122 (4), pp.3915-3934. 10.1002/2017JA023898 . insu-01522857

\section{HAL Id: insu-01522857 https://hal-insu.archives-ouvertes.fr/insu-01522857}

Submitted on 16 May 2017

HAL is a multi-disciplinary open access archive for the deposit and dissemination of scientific research documents, whether they are published or not. The documents may come from teaching and research institutions in France or abroad, or from public or private research centers.
L'archive ouverte pluridisciplinaire HAL, est destinée au dépôt et à la diffusion de documents scientifiques de niveau recherche, publiés ou non, émanant des établissements d'enseignement et de recherche français ou étrangers, des laboratoires publics ou privés. 


\section{Journal of Geophysical Research: Space Physics}

\section{RESEARCH ARTICLE \\ 10.1002/2017JA023898 \\ Statistics of electric fields' amplitudes in Langmuir turbulence: A numerical simulation study}

Key Points:

- Systematic study of the properties of Langmuir turbulence-driven electron beams via bump-on-tail instabilities in unhomogeneous plasmas

- Pearson technique is used to classify the calculated probability distribution functions (PDFs) of the logarithm of

the waves' amplitudes

- Universal scaling parameters are found, with values not depending on the levels of the density fluctuations and of the Langmuir turbulence

Correspondence to:

A. Voshchepynets,

woschep@gmail.com

\section{Citation:}

Voshchepynets, A., A. Volokitin, V. Krasnoselskikh, and C. Krafft (2017), Statistics of electric fields' amplitudes in Langmuir turbulence: A numerical simulation study, J. Geophys. Res. Space Physics, 122, 3915-3934, doi:10.1002/ 2017JA023898.

Received 17 JAN 2017 Accepted 8 APR 2017 Accepted article online 17 APR 2017 Published online 22 APR 2017

○2017. American Geophysical Union. All Rights Reserved.

\author{
A. Voshchepynets ${ }^{1,2}$ D, A. Volokitin ${ }^{3,4}$, V. Krasnoselskikh ${ }^{1}$, and C. Krafft ${ }^{5}$ \\ ${ }^{1}$ Laboratoire de Physique et Chimie de I'Environnement et de I'Espace (LPC2E), Orléans, France, ${ }^{2}$ Swedish Institute of \\ Space Physics, Kiruna, Sweden, ${ }^{3}$ Space Research Institute, Moscow, Russia, ${ }^{4}$ IZMIRAN, Moscow, Russia, ${ }^{5}$ LPP, CNRS, Ecole \\ polytechnique, UPMC Univ. Paris 06, Univ. Paris-Sud, Observatoire de Paris, Université Paris-Saclay, Sorbonne Universités, \\ PSL Research University, Palaiseau, France
}

Abstract A systematic study of the properties of Langmuir turbulence generated by electron beams via bump-on-tail instabilities in strongly nonhomogeneous plasmas is presented. A statistical analysis of the Langmuir waves' amplitudes using numerical simulations based on two theoretical models is performed: a dynamical one and a probabilistic one. The former describes the self-consistent dynamics of wave-particle and wave-wave interactions. The latter is a modified version of the quasi-linear theory. To analyze the simulation data provided by the probabilistic model, a Pearson technique is used to classify the calculated probability distribution functions (PDFs) of the logarithm of the waves' amplitudes. It is demonstrated that the core parts of the PDFs belong to the Pearson types I, IV, and VI distributions, while the high-amplitude parts of the PDFs follow power law or exponential decay. Analysis of the PDFs calculated using the numerical simulations based on the dynamical model leads to the following additional results. In the small-amplitude parts of the PDFs, a universal scaling parameter is found, with a value not depending on the average levels of the density fluctuations and of the Langmuir turbulence. Second, the PDFs are obtained in the presence of wave decay processes. When those are weak, the PDFs show at large fields' amplitudes an exponential asymptotic behavior; during time evolution, the corresponding scaling parameter decreases until a universal probability distribution is reached, indicating that the wave decay processes are sufficiently strong. Such exponential type of distribution is a specific signature of transition states in the Langmuir turbulence.

\section{Introduction}

The study of the statistics of electric fields' amplitudes became a more and more popular tool in the investigation of Langmuir wave turbulence in space plasmas. For instance, the statistics of electric fields' measurements were presented in cusp regions [LaBelle et al., 2010], in the vicinity of the Earth electron foreshock [Bale et al., 1998; Kellogg et al., 1999], and in the solar wind [Li et al., 2010; Reid and Kontar, 2017]. Small-amplitude waves present distributions related to linear instability properties, while the large-amplitude waves' statistics is influenced by the presence of nonlinear processes. Then the question to solve is whether it is possible to characterize quantitatively the role of linear and nonlinear effects at work in the Langmuir turbulence by analyzing probability distribution functions of the electric fields' amplitudes and to determine the influence of the density fluctuations' profiles on these distributions.

The interpretation of the statistics of the amplitudes' distributions often involves the Stochastic Growth Theory (SGT) [Robinson, 1995; Cairns and Robinson, 1999; Cairns and Menietti, 2001]. Initially, the SGT was developed to explain the properties of the Langmuir waves observed within the regions of solar type III radio bursts. In the standard model of type III radio bursts [Ginzburg and Zhelezniakov, 1958], the Langmuir waves are generated via a beam instability of the electrons streaming outward from the Sun. From the very beginning it was pointed out that the Langmuir waves are clumped into spikes with peak amplitudes typically 3 orders of magnitude above the mean. Smith and Sime [1979] proposed an explanation of the clumping phenomenon based on the idea that the plasma is inhomogeneous and that in most regions where the beam could excite the waves the characteristic scale of the inhomogeneity is comparable with the spatial growth rate. On this basis, Robinson [1992] proposed a model that describes the interaction of an electron beam with Langmuir waves in such inhomogeneous plasmas. The beam propagates alternatively through regions where the wave growth rate is alternatively positive and negative and, as a result of its interactions with the waves, the slope of 
the electron velocity distribution function oscillates close to the level of marginal stability. In turn, the resulting wave's growth rate has the form of a random walk in logarithm of the wave energy density, with an upper boundary approximately equal to the kinetic energy density of the beam. The proposed model allowed to explain the observed clumping phenomenon and make predictions regarding the probability distributions of the magnitudes of the clumps. Due to the central limit theorem, the SGT predicts a log-normal distribution for the amplitudes of the Langmuir waves' electric fields.

Event studies using different experimental data registered on board various spacecraft and in laboratory plasmas [Cairns and Robinson, 1999; Cairns and Menietti, 2001; Austin et al., 2007] found distributions of amplitudes close to the log-normal. However, different nonlinear physical processes, such as the electrostatic decay of Langmuir waves, can be the cause of deviations of the distributions from the log-normal. For instance, close to the foreshock boundary [Cairns and Menietti, 2001] and averaged over the foreshock [Bale et al., 1997; Cairns and Robinson, 1997], the distributions appear to be power laws at high fields $E$; i.e., $P(E) \sim E^{-2}$. The spatial variations of the waves' parameters and the relatively small number of fields' samples and periods analyzed made it difficult to assess deviations from log-normal distributions in these analyses. An additional observational evidence of the role of nonlinear processes for the large-amplitude waves' statistics was presented by Soucek et al. [2005].

The statistics of the observed electric fields' amplitudes can also be determined by wave scattering (diffusion in the wave phase velocity) on the background plasma density fluctuations. Recently, Krasnoselskikh et al. [2007] proposed a model that describes the linear interaction of Langmuir wave packets with an electron beam and takes into account two important effects caused by the density irregularities, namely the angular diffusion of the wave vectors due to wave scattering on small-amplitude density fluctuations and the suppression of the beam instability caused by the removal of the waves from their resonance conditions with the particles when crossing density perturbations of relatively large amplitudes. The authors showed that, under some circumstances, distributions of the logarithm of the wave intensity can belong to Pearson type IV distributions rather than to normal ones. The main reason for the deviations of the distributions from the normal is that the effective number of regions where the waves grow is not very large and, as a consequence, the central limit theorem fails to be true.

We study here the probability distribution functions (PDFs) of the waves' electric fields calculated by two kinds of numerical simulations of the Langmuir turbulence excited by weak electron beams in inhomogeneous plasmas. The first kind is based on a probabilistic model of beam-plasma interaction mentioned above [Voshchepynets et al., 2015; Voshchepynets and Krasnoselskikh, 2015] (see also Appendix A) and the second one on a self-consistent dynamical model involving wave-particle as well as wave-wave interactions [Krafft et al., 2013] (see also Appendix B). The latter one allowed to efficiently study various physical problems, concerning nonlinear and turbulent stages of different instabilities of electron or ion distributions, wave-particle interactions at multiple resonances [Krafft et al., 2005; Krafft and Volokitin, 2006; Zaslavsky et al., 2006, 2007; Krafft et al., 2010], quasi-linear diffusion processes of particles in wave packets [Volokitin and Krafft, 2012], wave turbulence in randomly inhomogeneous plasmas, wave scattering, reflection and decay, etc. [Krafft et al., 2015]. Recently, the dynamical model was used to study the resonant interaction of an electron beam with Langmuir wave packets in plasmas with random density fluctuations, under conditions typical of solar type III bursts regions [Volokitin et al., 2013; Krafft et al., 2013, 2014]. Comparison of the waveforms of the Langmuir waves obtained with recent measurements by the STEREO and Wind satellites showed that their characteristic features are very similar [Krafft et al., 2014]. Moreover, the model was used to study the statistics of Langmuir waves when the decay instability is developed as well as particle acceleration and diffusion processes at work in the fluctuating plasma [Krafft and Volokitin, 2016; Volokitin and Krafft, 2016]. On the other hand, the probabilistic model, which is a modified version of the quasi-linear theory of the weak turbulence in the randomly inhomogeneous plasma, requires much less computational resources. Thus, it enables us to perform a detailed analysis of the statistics of the amplitudes of the Langmuir wave fields.

The purpose of our study is to determine as precisely as possible the type of distributions that describe the PDFs calculated in our simulations. Moreover the dependence of the Langmuir fields' distributions on the spatial profiles of the density fluctuations is of particular interest. To this end we use a powerful method proposed by Pearson [1895], which allows to perform a classification of the distributions according to their first four statistical moments, each class corresponding to well-known distributions. In this frame, the cores and the high-amplitude parts of the PDFs will be interpolated to find some universal fitting parameters. In the first 
part, we describe the results obtained with the probabilistic model, whereas the second part of the paper is devoted to the analysis of the simulations provided by the dynamical model, including the effects of Langmuir wave decay on the calculated PDFs.

\section{PDFs of the Electric Fields' Amplitudes: Probabilistic Model}

The recently developed probabilistic model might provide an alternative approach to the problem of beam-driven Langmuir turbulence in a fluctuating plasma. It allows to estimate statistically the averaged rate of energy exchange between waves and particles interacting at Landau resonance. Using a procedure of averaging, it takes into account the fact that the phase velocity of a Langmuir wave undergoes variations as the wave propagates within the fluctuating plasma. For the sake of logical completeness, we present hereafter a brief summary of the model.

To apply this statistical approach to the system, we replace the continuous spatial interval by a discrete one of finite (but very large) length and divide it into a set of equally sized and small subintervals $\Delta x$. Then let us consider the interaction of a small-amplitude coherent wave with an electron within $\Delta x$, assuming that the density profile is linear along $\Delta x$. Our assumptions allow one to describe the action of a wavefield of known frequency on a particle with the sufficient accuracy and to calculate the effects of the wave-particle interactions occurring along any $\Delta x$ with chosen densities at boundaries. The key point of our description is that the values of the density at the center of each subinterval $\Delta x$ are assumed to be random and independent and that they are described by a predetermined known statistical distribution. This in turn allows us to introduce a new important function $P_{\omega_{i}}(V)$ that describes the probability that a wave of frequency $\omega_{i}$ has a phase velocity $V$ on a certain subinterval $\Delta x$; this probability is directly dependent on the distribution of the amplitudes of the density fluctuations. The probability function $P_{\omega_{i}}(V)$ allows one to calculate the average energy exchange between particles of given velocities and a wave of given frequency. Note that the wave-particle interactions occurring along each subinterval are supposed to be independent of those occurring along previous subintervals. Using such assumptions, we calculate the probability that a particle of velocity $v_{0}$ at initial time $t_{0}$ will have, after $Q$ interactions occurring during a time interval $t-t_{0}$, a velocity $v$, where $Q$ should be large enough to justify a statistical averaging. The assumption of random and independent interactions corresponds to an uncorrelated Markovian process and leads to a description of the evolution of the electron velocity distribution function based on an equation of diffusion similar to the Fokker-Planck equation in the velocity space. Under such assumptions, the diffusion coefficient determined is dependent on $P_{\omega_{i}}(V)$ and, as a result, on the probability distribution of the density fluctuations. To calculate the growth rate of a wave, we use the variation of the particles' energy and take into account the fact that, on a small subinterval, the gain/loss of energy of a particle is equal to the energy lost/gained by the wave.

In some sense the model generalizes the well-known quasi-linear $(\mathrm{QL})$ theory to the case of nonuniform plasmas with random density fluctuations. With known $P_{\omega_{i}}(V)$ the equations of evolution of the wave spectrum and the electron velocity distribution function can be readily presented in a form close to the QL theory (see the equations (A1) and (A2) in Appendix A). For a more detailed description we refer to the original papers [Voshchepynets et al., 2015; Voshchepynets and Krasnoselskikh, 2015] as well as to Appendix A.

If a pure probabilistic model does not provide any information regarding the spatial distributions of the Langmuir electric fields, it is not so for the energy density of the waves. By making use of the WKB approximation, the electric field of a monochromatic Langmuir wave with frequency $\omega_{i}$ and wave vector $k_{i}(x)$ is given by [Krasnoselskikh et al., 2007]

$$
E_{i}(t, x)=\frac{\sqrt{8 \pi W_{i}}}{k_{i}} \exp \left(-i \omega_{i} t+i k_{i}(x) x+\psi_{i}\right),
$$

where $W_{i}$ is the wave energy density (see Appendix A for details), $k_{i}(x)$, the wave vector that undergoes spatial changes due to the density fluctuations, and $\psi_{i}$, the initial phase. Equation (1) takes into account the fact that the field amplitude can vary during the wave propagation because the group velocity can change. On its turn, the determination of the spatial distribution of $k_{i}(x)$ requires to know the spatial profiles of the plasma density. Thus, the spectrum of the density fluctuations has to be studied in detail by means of different techniques.

It is known from in situ spacecraft measurements in the solar wind [Neugebauer, 1975; Celnikier et al., 1987; Kellogg and Horbury, 2005; Chen et al., 2012] that the density spectrum within the frequency domain $10^{-2}-10^{1} \mathrm{~Hz}$ can be considered as a two-knee power law with a breaking frequency around $0.6 \mathrm{~Hz}$. Part of the 
spectrum below the break can be approximated quite well using the Kolmogorov power law with a spectral index of $-5 / 3$. The spectral index above the break depends on the conditions of the solar wind; nevertheless, its value typically lies in the range $[-0.91,-0.38$ ] [Celnikier et al., 1987]. It is worth noting that other authors found different breaks in the spectrum. A comparison of earlier measurements of the density fluctuation spectra can be found in Figure 5 of Kellogg and Horbury [2005], who found that, depending on the average density, the spectrum can be well described by a single power law with a spectral index of -1.37. Recently, Chen et al. [2012] reported about a spectral index of -2.7 above the break, based on the Artemis data.

\subsection{Reconstruction of the Spatial Profiles of the Langmuir Waves}

Our purpose is to reconstruct time series of density fluctuations with spectral properties similar to those observed in the solar wind plasmas. For this aim we use a technique analogous to that described by Kellogg et al. [1999]. First, we divide the density power spectrum into $10^{4}$ equally sized intervals and calculate series of coefficients $A_{i}$ equal to the square root of the power spectrum multiplied by the width of one interval. For this procedure we use a spectrum similar to that reported by Celnikier et al. [1987], with a spectral index of -0.61 above the break. Then, the coefficients $A_{i}$ allow us to reconstruct synthetic density data as follows:

$$
n(t)=\sum_{i} A_{i} \cos \left(\omega_{i} t+\phi_{i}\right)
$$

where $\phi_{i}$ is a random phase which is assumed to be uniformly distributed in $[0,2 \pi]$. Different initial sets of $\phi_{i}$ lead to various time series of density variations. To obtain the spatial profiles of the plasma density, we use the Taylor hypothesis and get the spatial dependence of the density fluctuations taking into account that fluctuations propagate with the solar wind velocity, i.e., $n(x)=n\left(v_{\mathrm{sw}} t\right)$ and $x=x_{0}+v_{\mathrm{sw}} t$, where $v_{\mathrm{sw}}$ is the speed of the solar wind. Two examples of such synthetic spatial profiles are shown in Figure 1.

During the propagation of a Langmuir wave within the fluctuating plasma, its wave vector undergoes variations. The relationship between $n(x)$ and $k_{i}(x)$ can be found from the nonlinear dispersion equation, under the assumption that the wave frequency $\omega_{i}$ remains constant:

$$
\omega_{i}=\omega_{p 0}\left(1+3 k_{i}^{2} \lambda_{D}^{2} / 2+\frac{1}{2}\left(1-\frac{n(x)}{n_{0}}\right)\right),
$$

where $n_{0}$ and $\omega_{p 0}$ are the density and the plasma frequency of the unperturbed plasma, respectively, and $\lambda_{D}$ is the Debye length.

The available synthetic data sets of $n(x)$ enable us to reconstruct $k_{i}(x)$ for any Langmuir wave with known $k_{i}\left(x_{0}\right)$ at point $x_{0}$ that satisfies $n\left(x_{0}\right)=n_{0}$. Then the spatial profiles of the wave fields of the Langmuir packets can be obtained using equation (1). Two profiles $|E(x)|^{2}=\left|\sum_{i} E_{i}(x)\right|^{2}$ are shown in Figure 1, for which a set of 2000 monochromatic Langmuir waves with frequencies lying within the range $\left[1.0001 \omega_{p 0}, 1.0625 \omega_{p 0}\right]$ have been used. The field amplitudes $E_{i}(x)$ are calculated from the wave energy densities $W_{i}$ shown in Figure 14 (left), in the time interval where the total energy density $W_{t}$ reaches its maximum Figure 14 (right).

We have found that the spatial distributions $|E(x)|^{2}$ strongly depend on the exact forms of the spatial profiles of the density fluctuations. Despite the fact that the fluctuations $\delta n / n_{0}=\left(n(x)-n_{0}\right) / n_{0}$ shown in Figure 1 have identical averaged characteristics, i.e., $\left\langle\left(\delta n(x) / n_{0}\right)^{2}\right\rangle_{x}^{1 / 2}=0.02$ (note that the averaging was performed over a spatial interval hundred times larger than that shown in Figure 1), the reconstructed spatial distributions of the electric fields show different qualitative features. The profile $|E(x)|^{2}$ shown in Figure 1 (top) is rather uniformly distributed in space, while a localized clumpy structure is clearly recognizable in that one presented in Figure 1 (bottom). It is worth noting that the magnitude of the electric field within the clump is typically 10 times larger than the maximum of $|E(x)|$ observed in Figure 1 (top).

After having analyzed more than 100 different density profiles, we found that the profiles containing relatively large positive deviations from the background density, i.e., $\delta n / n_{0} \geq 0.01$, are preferable for the formation of the clumps. In this case the local plasma frequency $\omega_{p}(x) \simeq \omega_{p 0}\left(1+\delta n / 2 n_{0}\right)$ is close to the frequencies $\omega_{i}$ of the Langmuir waves chosen for the simulations. Then, as $\omega_{i}-\omega_{p}(x) \rightarrow 0$, the local wave vector $k_{i}(x)$ tends to zero to satisfy the nonlinear dispersion relation (3). The smallness of the wave vector leads to two notable effects, namely, the localization of the wave packet in the vicinity of $x$ and the amplification of its amplitude. The first effect appears as a result of the vanishingly small term $k_{i}(x) x$ in equation (1) and the second one is 

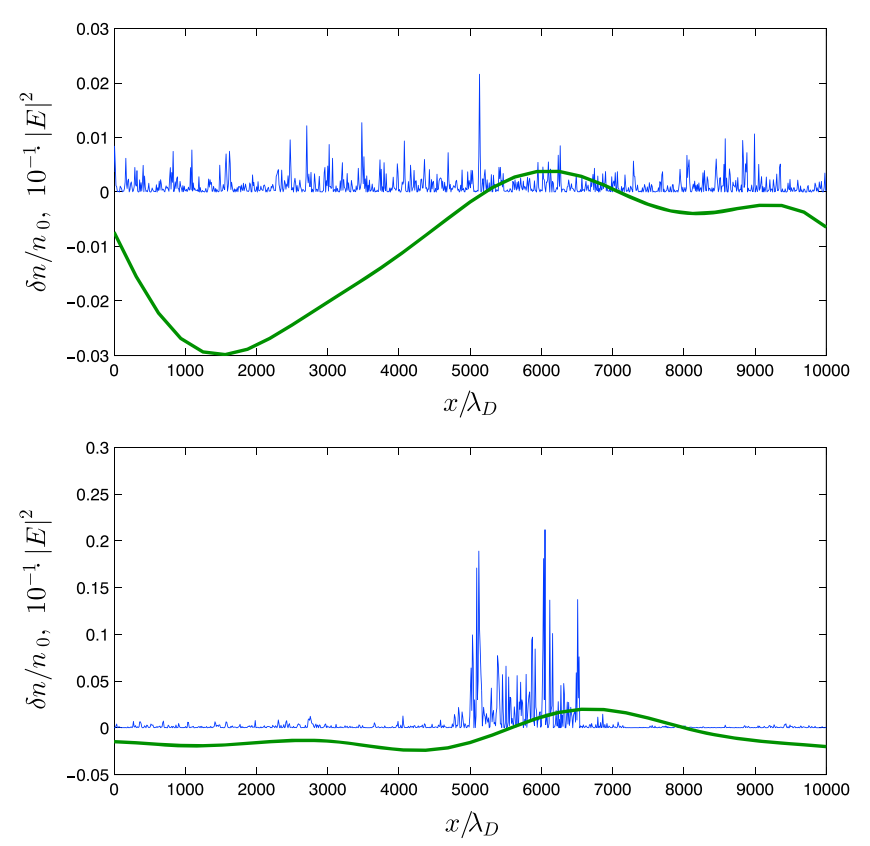

Figure 1. (top and bottom) Spatial distributions of the density fluctuations $\delta n / n_{0}=\left(n(x)-n_{0}\right) / n_{0}$ (in green) superimposed with the spatial distributions of the energy density $|E(x)|^{2}$ of the Langmuir wave packet (in blue). The average level of the density fluctuations is $\Delta n=0.02$.

related to the variation of the wave group velocity $v_{\mathrm{gr}}=3 k_{i}(x) \lambda_{D}$. As $k_{i}(x) \rightarrow 0$, the group velocity also tends to 0 , which leads to the localization of the wave energy in the vicinity of the point $x$. To avoid singularities, we exclude from our consideration the spatial profiles of density fluctuations that contain points $k_{i}(x)=0$.

\subsection{Probability Distributions of the Amplitudes of the Langmuir Waves' Fields}

We performed simulations in plasmas with different average levels of density fluctuations; i.e., $\Delta n=$ $\left\langle\left(\delta n / n_{0}\right)^{2}\right\rangle^{1 / 2}=0.01,0.02,0.03$, and 0.04 . For each $\Delta n$, we generated 30 different density profiles by using the procedure described above, in order to reconstruct the spatial profiles of the Langmuir electric fields. Following the SGT [Robinson et al., 1993] that predicts normal distributions for the waves' growth rates and thus log-normal distributions for the fields' amplitudes, we consider below the distributions of $\ln |E|^{2}$ instead of $|E|^{2}$. Examples of the calculated distributions are shown in Figure 2.

We make a distinction between the synthetic data that contain clumpy structures (Figure 1, bottom) and those in which such structures are not observed (Figure 1, top). Hereinafter, the distributions obtained from the first (respectively, second) type of data are referred to as $P_{s}\left(\ln |E|^{2}\right)$ (respectivelym $P_{l}\left(\ln |E|^{2}\right)$ ), whereas those corresponding to all available data are indicated by $P_{t}\left(\ln |E|^{2}\right)$. Histograms representing the distributions of the synthetic data are shown in Figure 2 by solid lines. To build them, we used $K_{b}=50$ bins with centers uniformly distributed from $\min \left(\ln \left|E_{i}\right|^{2}\right)$ to $\max \left(\ln \left|E_{i}\right|^{2}\right.$ ), where the values $E_{i}$ are the reconstructed amplitudes of electric fields obtained using simulations with the same level of density fluctuations. It should be noted that the results obtained correspond to the stage of well-developed turbulence.

Figure 2 shows the PDFs obtained for $\Delta n=0.01$ (top) and $\Delta n=0.03$ (bottom). To build $P_{t}\left(\ln |E|^{2}\right.$ ), we first calculate the distributions of $\ln |E|^{2}$ using the $E_{i}^{2}$ collected on each of the 30 density profiles and then make averaging. Thus, $K_{t} P\left(\ln \left|E_{i}\right|^{2}\right)$ is the averaged number of $\ln \left|E_{i}\right|^{2}$ in the ith bin, where $K_{t}$ is the total number of $E_{i}$ values reconstructed on one density profile. To obtain $P_{s}\left(\ln |E|^{2}\right)$ and $P_{I}\left(\ln |E|^{2}\right)$, averaging is performed over the number of density profiles corresponding to the first and the second types of data, respectively.

Due to the central limit theorem, distributions close to the normal one are often found in nature. Our purpose is to determine the distributions that can describe the data obtained in our simulations as precisely as possible. To perform the analysis of the calculated distributions, we use a technique proposed by Pearson [1895], which allows to classify continuous probability distribution functions according to their first four statistical moments $\mu_{i}, i=1$, 4. Pearson showed that, depending on the parameters $\beta_{1}=\mu_{3}^{2} / \mu_{2}^{3}$ and $\beta_{2}=\mu_{4} / \mu_{2}^{2}, 12$ types of 

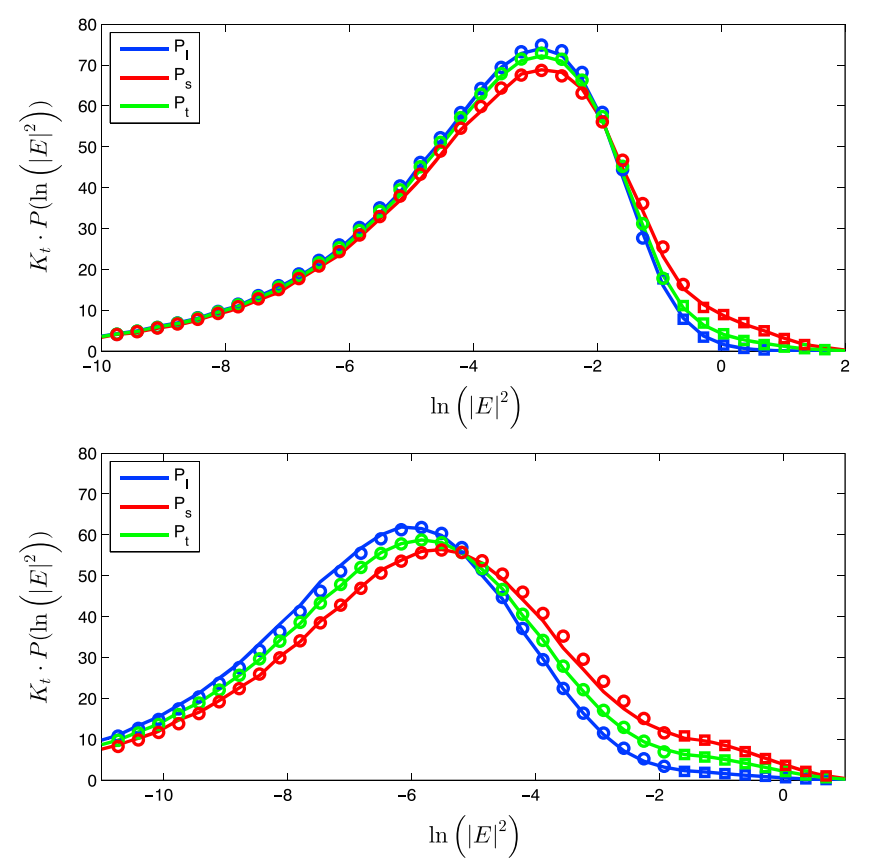

Figure 2. Probability distributions $P\left(\ln |E|^{2}\right)$ of the logarithm of the amplitudes of the Langmuir waves' fields, calculated by the simulations. (top) $\Delta n=0.01$; (bottom) $\Delta n=0.03$. The different colors correspond to the different data sets: green, all available data are used; red, only data containing recognizable clumps are used; blue, data without recognizable clumpy structures are used. Solid lines correspond to the observed distributions; circles, fits of the core distributions with Pearson curves; squares, fits of the high-amplitude tails.

distributions might be distinguished, including such famous distributions as the normal $\left(\beta_{1}=0, \beta_{2}=3\right.$, Pearson type VII), the exponential ( $\beta_{1}=9, \beta_{2}=4$, Pearson type X), the gamma (Pearson type III), and the beta distributions (Pearson types I and II). All 12 types are summarized together with their analytical form in Table 1 of Podladchikova et al. [2003]. It is worth noting that, if at least one of the first four moments does not exist, the considered distribution function cannot be classified by the proposed technique.

Analysis of the obtained distributions shows that $P_{t}\left(\ln |E|^{2}\right)$ corresponds to a Pearson type IV or type VI distribution, depending on the average level $\Delta n$. For instance, the distribution obtained for $\Delta n=0.01$ (Figure 2, top) is characterized by $\beta_{1}=1.8$ and $\beta_{2}=6.5$ and, according to the Pearson classification, it corresponds to the type VI [Podladchikova et al., 2003], follows a beta prime distribution (also known as the inverted beta distribution) and depends on three parameters that can be determined from the moments $\mu_{i}$. The fit of $P_{t}\left(\ln |E|^{2}\right)$ by a Pearson type VI distribution is marked by green circles in Figure 2 (top). The fitting curve was obtained by using the statistical moments $\mu_{i}$ calculated directly from the data sets and by applying the Levenberg-Marquardt method [Press et al., 1992].

To evaluate the relevancy of the fits, we use a chi-square statistical test [Bendat and Piersol, 1980] which is based on the observation that, if the data follow a given distribution, the normalized error $X^{2}$ of the fit is a random variable that obeys a chi-square distribution $\chi_{v}^{2}$ with a degree of freedom $v=K_{b}-K_{f}-1$, where $K_{f}$ is the number of free parameters in the fitting function. Thus, the hypothesis that the data are distributed according to the predicted distribution can be tested by comparing the value of $X^{2}$ to a percentage point $\chi_{\alpha, v}^{2}$ of the chi-square distribution at a chosen significance level $\alpha$. The Levenberg-Marquardt method is based on the minimization of $X^{2}$ that allows to increase the significance level. For instance, $X^{2}=0.5$ for the PDF $P_{t}\left(\ln |E|^{2}\right)$ shown in Figure 2, which indicates that the hypothesis that the data follow a Pearson type VI distribution cannot be rejected even at a significance level above $99.9 \%$.

We have found that for the case of small levels of density fluctuations $(\Delta n<0.02)$ the data containing clumpy structures provide PDFs $P_{s}\left(\ln |E|^{2}\right)$ that obey Pearson type IV distributions (see the red curves in Figure 2), while those without these structures (i.e., $P_{I}\left(\ln |E|^{2}\right)$ ) correspond to Pearson type VI probability distributions (blue curves in Figure 2 ). To make the fit of the histogram of the first class of data (shown by red circles), we first calculate the moments $\mu_{1}-\mu_{4}$ (getting $\beta_{1}=1.4$ and $\beta_{2}=5.9$ ) and then use the general formula for a 


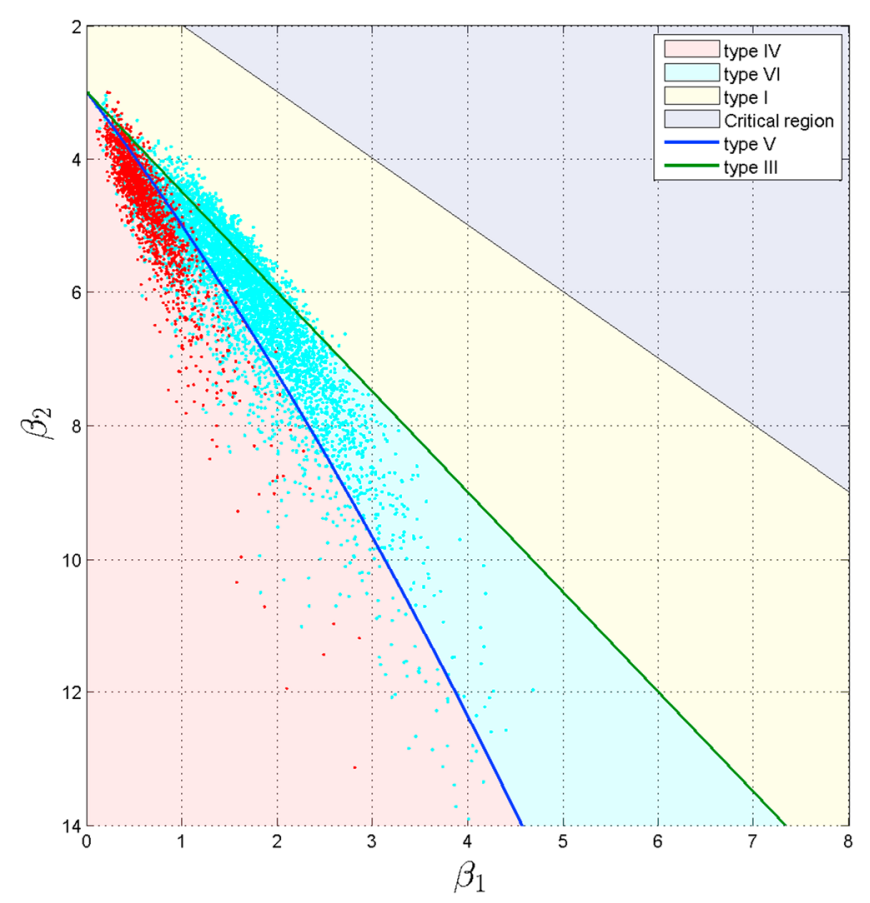

Figure 3. Scatterplot of the distributions of the logarithm of the Langmuir fields' amplitudes $|E|$ on the Pearson diagram; $\beta_{1}$ and $\beta_{2}$ are the Pearson parameters. Areas filled with different colors correspond to different types of Pearson distributions; certain distributions are represented by curves (see the legend). The average level of density fluctuations is $\Delta n=0.02$.

type IV distribution [Podladchikova et al., 2003]. Applying the method of maximum likelihood provides the normalized error $X^{2}=0.6$, indicating that the data follow the model distribution with a very high precision. The fit of the histogram of the second class of data by a Pearson type VI distribution $\left(\beta_{1}=2\right.$ and $\left.\beta_{2}=6.8\right)$ is shown by blue circles (with $X^{2}=0.7$ ).

The distribution functions obtained for $\Delta n=0.03$ are shown in Figure 2 (bottom). Contrary to the previous case $(\Delta n=0.01)$, the PDFs $P_{t}\left(\ln |E|^{2}\right), P_{s}\left(\ln |E|^{2}\right)$, and $P_{l}\left(\ln |E|^{2}\right)$ obey Pearson type IV distributions with the following sets of $\left(\beta_{1}, \beta_{2}\right)$ parameters: $(0.8,6.1),(0.45,6.81)$, and $(0.98,5.48)$, respectively; the corresponding fits are shown by green, blue, and red circles, respectively. Despite the fact that $P_{t}\left(\ln |E|^{2}\right), P_{s}\left(\ln |E|^{2}\right)$, and $P_{l}\left(\ln |E|^{2}\right)$ correspond to the same type of Pearson distributions, some differences exist between them: (1) $P_{s}\left(\ln |E|^{2}\right)$ has a larger dispersion $(\sigma=2.7)$ than $P_{I}\left(\ln |E|^{2}\right)$, for which $\sigma=2.5 ;(2) P_{s}\left(\ln |E|^{2}\right)$ has a higher expected value (indeed $\left\langle\ln |E|^{2}\right\rangle=-6.06$ for $P_{s}\left(\ln |E|^{2}\right)$ and $\left\langle\ln |E|^{2}\right\rangle=-6.80$ for $P_{/}\left(\ln |E|^{2}\right)$ ).

It is worth noting that the Pearson curves are suitable for fitting the cores of the obtained distributions. In the range of large amplitudes (typically above $\left\langle\ln |E|^{2}\right\rangle+2 \sigma$ ), the discrepancies between histograms and fitting functions are significant. Therefore, we exclude these high-amplitude parts from the consideration when fitting the cores of the distributions; their analysis shows that they can follow power law or exponential functions depending on the type of core distributions. We have found that when the core part follows a Pearson type VI distribution, the high-amplitude part follows a power law, $P\left(\ln |E|^{2}\right) \sim\left(|E|^{2}\right)^{-p_{p l}}$, with $p_{p l}$ typically lying within the range 1.48-2.51 (see the blue and green squares in Figure 2, top). On the contrary, when the core part corresponds to a Pearson type IV distribution, the high-amplitude part follows an exponential decay, $P\left(\ln |E|^{2}\right) \sim \exp \left(-p_{\text {ed }}|E|^{2}\right.$ ), with $1.29<p_{\text {ed }}<1.51$ (see the blue, green, and red squares in Figure 2, bottom).

Until now our study was focused on the aggregated distributions (histograms include data reconstructed by using different spatial profiles of density fluctuations). Beside this, we considered only those time intervals where the wave energy, gained from the electron beam during the process of relaxation, was about its maximum. Let us now consider separately each particular distribution describing the values $E_{i}\left(x_{j}\right)$ obtained from simulations with different density profiles and at different moments of time. For each time interval $\Delta t_{i}=t_{i}-t_{i-1}$ we use the corresponding wave energy density $W_{i}\left(t_{i}\right)$ obtained in the simulations to reconstruct the profiles of $E_{i}^{2}\left(x_{j}\right)$ at $\Delta t_{i}$ on an appropriate spatial scale (of length $10,000 \lambda_{D}$ ). One data set consists 

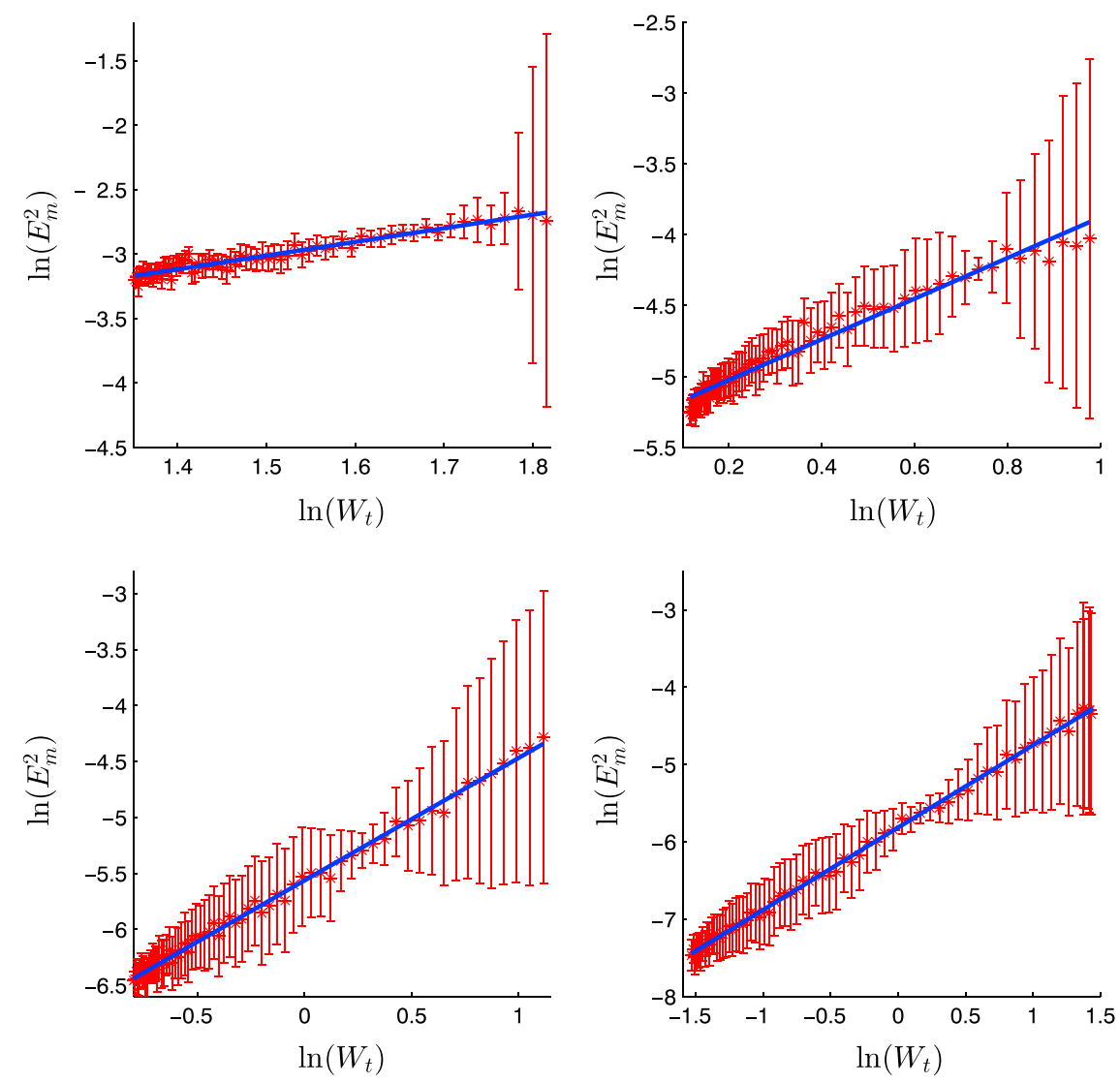

Figure 4. Dependence of $\ln \left(E_{m}^{2}\right)$ on the level of the Langmuir turbulence $W_{t}$. The points are marked by red asterisks and fits by blue lines. Simulations' results with different levels of density fluctuations are shown: (top left) $\Delta n=0.01$; (top right) $\Delta n=0.02$; (bottom left) $\Delta n=0.03$; (bottom right) $\Delta n=0.04$.

of $j=1024$ values of $E_{i}\left(x_{j}\right)$ collected on such a spatial interval. For each data set, we calculate the statistical moments $\mu_{1}-\mu_{4}$ and then use the Pearson technique to classify the corresponding distributions by making use of the Pearson's betas. Figure 3 shows the results obtained for $\Delta n=0.02$ as a scatterplot in the $\beta_{1}-\beta_{2}$ plane. The areas with different colors correspond to different types of distributions: red area, Pearson type IV; green area, Pearson type Vl; yellow area, Pearson type I. Certain distributions are represented with curves: blue line, Pearson type $V$ distribution; green line, Pearson type III. It is worth noting that the normal distribution is represented by a single point with coordinates $\beta_{1}=0$ and $\beta_{2}=3$.

As previously, we made a distinction between data that contain clumps and data without such structures. The first (respectively, second) class is represented by red (respectively, cyan) points. The distributions obtained using the data with clumpy structures are concentrated in the domains $0 \leqslant \beta_{1} \leqslant 1$ and $3 \leqslant \beta_{2} \leqslant 5$. Most of them correspond to the Pearson type IV distribution, except for a small number of them that correspond to types I, V, and VI. The distributions obtained using the second class of data are characterized by a wider range of parameters: $2 \leqslant \beta_{1} \leqslant 3$ and $4 \leqslant \beta_{2} \leqslant 8$. The most common distributions, which constitute approximately $70 \%$ of those obtained from the second class of data, correspond to Pearson type VI distributions. Types I and III distributions are also often observed and constitute slightly less than $25 \%$ of the total amount. Analysis of the data obtained in the simulations with larger $(\Delta n=0.03-0.04)$ as well as lower $(\Delta n=0.01)$ levels of density fluctuations provide the same results: $P\left(\ln |E|^{2}\right)$ follows Pearson distributions of types IV and $V$ when the data contain clumpy structures and Pearson distributions of types I, III, and VI in the opposite case.

In addition, we have studied the dependence of the PDFs of the reconstructed electric fields on the level of the Langmuir turbulence. For this purpose we use both the energy density $W_{t}\left(t_{i}\right)$ calculated in the simulations and the distributions of the electric fields reconstructed from the different spatial profiles of density fluctuations. We consider only time periods after the wave energy density has reached its maximum (e.g., for $t>10$ au, Figure 14, right). To each time interval $\Delta t_{i}$ corresponds one value of $W_{t}\left(t_{i}\right)$ and a set of 30 histograms 
$P_{i}\left(\ln |E|^{2}\right)$. For each of them, we determine the value $\ln \left(|E|_{m}^{2}\right)_{i}$ at which the maximum of $P_{i}\left(\ln |E|^{2}\right)$ is reached and then calculate the corresponding averaged value $\ln \left(E_{m}^{2}\right)$ in order to study its dependence on the level of the Langmuir turbulence $W_{t}\left(t_{j}\right)$. The results are shown by red asterisks in Figure 4 , for different levels of density fluctuations, the blue lines represent the least squares fits.

One can see that $\ln \left(E_{m}^{2}\right)$ is linearly dependent on $\ln W_{t}\left(t_{i}\right)$, with a coefficient of proportionality around $\alpha \simeq 1$, for all levels of density fluctuations except $\Delta n=0.02$ (Figure 4, top right) for which $\alpha \simeq 1.29$. When the average level of density fluctuations is relatively low (e.g., $\Delta n=0.01$ ), the relaxation evolves as in a homogeneous plasma; i.e., $W_{t}\left(t_{i}\right)$ only slightly decreases after it reaches its maximum. As a result, the values of $\ln \left(E_{m}^{2}\right)$ are observed in a narrow range of values (e.g., see Figure 4 , top left). Increasing $\Delta n$ leads to the broadening of the resonance conditions of the wave-particle interactions that makes the process of wave energy reabsorption by the energetic electrons very efficient. The energy of the wave can decrease several times (see Figure 14, right) after reaching its maximum, leading to the broadening of the range of $\ln \left(E_{m}^{2}\right)$.

It is worth noting that the dispersion of $\ln \left(E_{m}^{2}\right)$ (shown as error bars in Figure 4) increases with $\ln W_{t}\left(t_{i}\right)$. As already seen (Figure 2, bottom), the presence of localized structures of large amplitudes in the data results in the shift of $\ln \left(E_{m}^{2}\right)$ toward larger values. In the proposed model, clumps appear only on the spatial profiles of density fluctuations that contain large positive deviations from the background plasma density. Their number is around $12 \%$ of the total number of density profiles used for the reconstruction of the electric fields. Thus, the large-amplitude structures are present in the data relatively rarely. This might be the explanation of the large dispersion in the region of $\operatorname{large} \ln \left(E_{m}^{2}\right)$.

\section{PDFs of the Electric Fields' Amplitudes: Dynamical Model}

This section presents typical probability distributions of the amplitudes $|E|$ of the Langmuir electric fields calculated by 1-D numerical simulations based on the so-called dynamical model mentioned in section 1 and summarized in Appendix B, which is aimed to study the Langmuir turbulence generated by electron beams in inhomogeneous plasmas as the solar wind [Krafft et al., 2013]. It provides the opportunity to study many physical effects, as the acceleration of the high-energy part of the electron beam due to scattering or transformation of Langmuir waves on the background density fluctuations [Krafft and Volokitin, 2016], the formation of localized wave packets with waveforms similar to those observed by satellites (wave clumping phenomenon) [Krafft et al., 2014], or the development of Langmuir waves' decay competing with wave reflection and refraction phenomena [Krafft et al., 2015].

Concerning the study of the statistics of the electric fields' amplitudes and the influence of the background density fluctuations on the corresponding PDFs, it is worth to compare the results of the simulations based on the dynamical model with those obtained above within the framework of the probabilistic model. Both approaches have their advantages and disadvantages; whereas the spatial distributions of the electric fields in the former model can be obtained directly, their calculations in the frame of the latter one require to perform a special field reconstruction according the above described procedure. On the other hand, if performed with large simulation boxes containing a lot of wave packets and density fluctuations, as required ideally for a relevant statistical analysis, the dynamical model needs much more computational resources.

Figure 5 shows the time evolution of the electron beam-Langmuir waves' system, obtained for a low average level of density fluctuations, i.e., $\Delta n \leq 0.001$. One can see the time variation of the total wave energy density $W_{L}$, the spatial profiles of the Langmuir wave envelope $E$, and of the density fluctuations $\delta n / n_{0}$ at $\omega_{p 0} t=70,000$, as well as the beam velocity distributions $f(v)$ at $\omega_{p 0} t=0$ and $\omega_{p 0} t=70,000$. The rapid fluctuations visible in the profile of $\delta n / n_{0}$ (which are superposed to the long-wavelength density inhomogeneities) appear as a result of wave decay instabilities, when beam-driven Langmuir waves decay in backscattered Langmuir waves and ion acoustic waves [Krafft et al., 2013, 2015]. As expected when $\Delta n$ is sufficiently low, the dynamics of the system evolves in accordance with the expectations of the quasi-linear theory (wave saturation, beam relaxation with plateaued velocity distribution, etc. [Krafft et al., 2013]).

In order to determine the PDFs of the electric fields' amplitudes within given ranges of space and time, we divide the time interval $\Delta T$ selected for the analysis into several subintervals; within each of them we calculate the number of cases when the amplitude $\left|E\left(t_{i}, x_{j}\right)\right|$ of the field at position $x_{j}$ and time $t_{i}$ falls within a small given interval $[E+\Delta E, E]$. Finally, we average the obtained distributions on all the time subintervals to get the final PDF presented in Figure 6. Note that hereinafter, nonnormalized PDFs are considered. For a suitable 

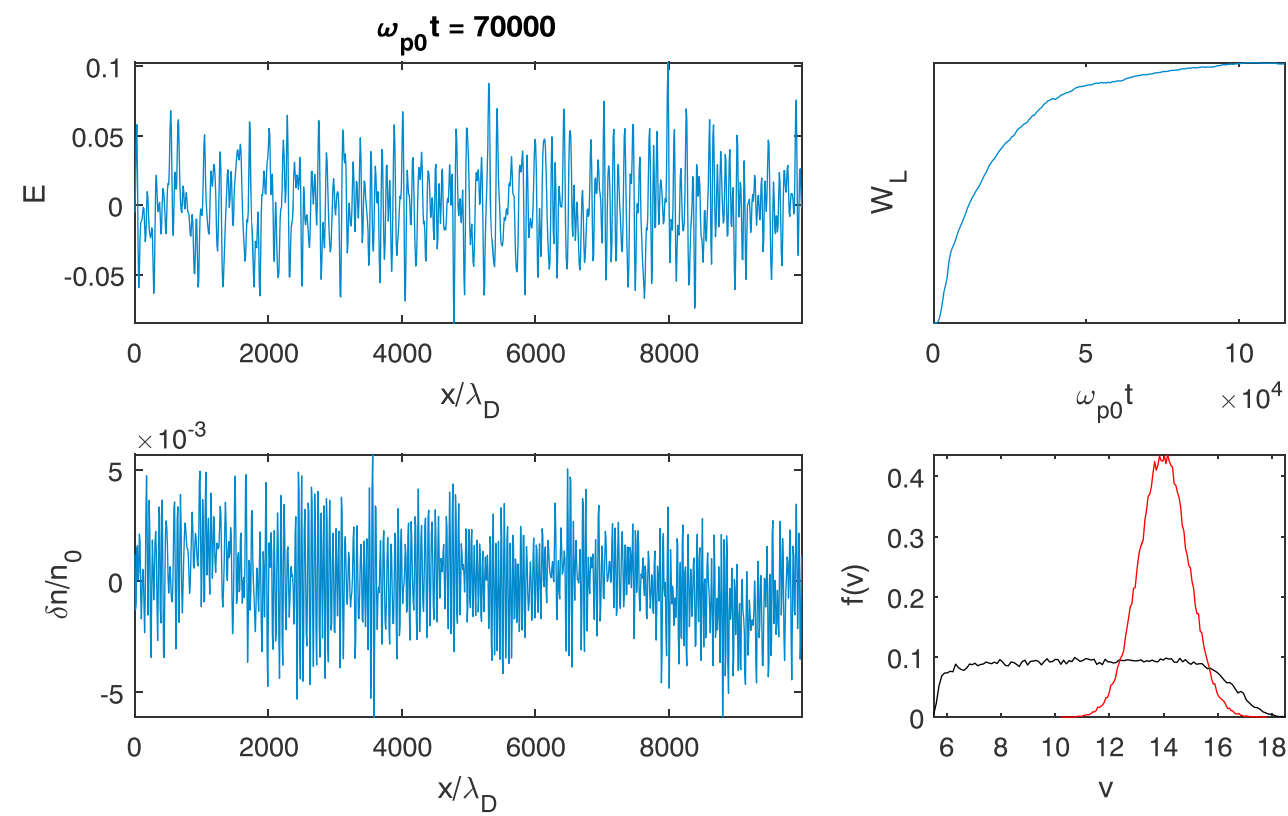

Figure 5. (left column) Space profiles of the Langmuir field envelope $E$ and of the density fluctuations $\delta n / n_{0}$, at time $\omega_{p 0} t=70,000$. (right column) Time evolution of the Langmuir wave energy density $W_{L}$ and of the beam velocity distributions $f(v)$ at $\omega_{p 0} t=0$ (Maxwellian) and $\omega_{p 0} t=70,000$ (relaxed plateau). The velocity $v$ is normalized by the plasma electron thermal velocity. The average level of density fluctuations is $\Delta n \leq 0.001$ (quasi-homogeneous plasma). The ratio of the beam to the plasma density is $n_{b} / n_{0}=0.00005$.

comparison with the space observations and the above studies, we show the dependencies of the PDFs on $\log _{10}|E|^{2}$ (Figure 6, left) as well as on $|E|$ (Figure 6, right). Straight lines indicate the following linear interpolations: $P\left(\log _{10}|E|^{2}\right) \sim \alpha_{1} \log _{10}|E|^{2}\left(\alpha_{1} \simeq 2.3\right.$, left side of the PDF, Figure 6, left), $P\left(\log _{10}|E|^{2}\right) \sim \alpha_{2} \log _{10}|E|^{2}$ $\left(\alpha_{2} \simeq-9.7\right.$, right side of the PDF, Figure 6, left), and $P(|E|) \sim \exp \left(\beta|E| /|E|_{m}\right)(\beta \simeq-5.8$, Figure 6, right), where $|E|_{m}$ corresponds to the statistical maximum value of $|E|$; it can be estimated from the condition $P\left(|E|<|E|_{m}\right) \simeq 1$, that in the case of a nonnormalized PDF, it means that, virtually, there is no case where $|E|>|E|_{m}$.

Testing this procedure for different intervals of time and space shows that the shapes of the obtained PDFs have a weak dependence on the selected intervals, if those are chosen within the stage when the Langmuir turbulence is well developed. Such amplitudes' distributions were also obtained for other simulations with $\Delta n \leq 0.001$, exhibiting similar characteristics for the various values of $\Delta n$.
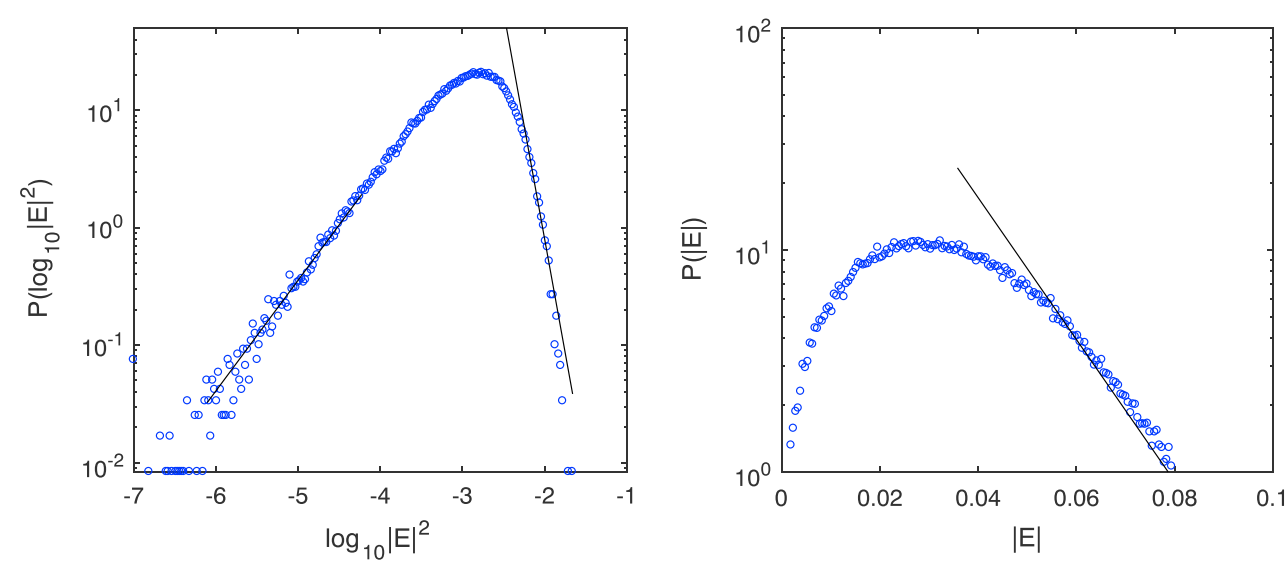

Figure 6. Averaged PDFs of the electric fields' amplitudes obtained for the simulation presented in Figure 5 , in the normalized time interval $\Delta T=[50,000-60,000]$. (left) Distribution of $\log _{10}|E|^{2}$, with linear fits' coefficients $\alpha_{1} \simeq 2.3$ and $\alpha_{2} \simeq-9.7$. (right) Distribution of $|E|$, in logarithmic scale, with the linear fit's coefficient $\beta \simeq-5.8$. 

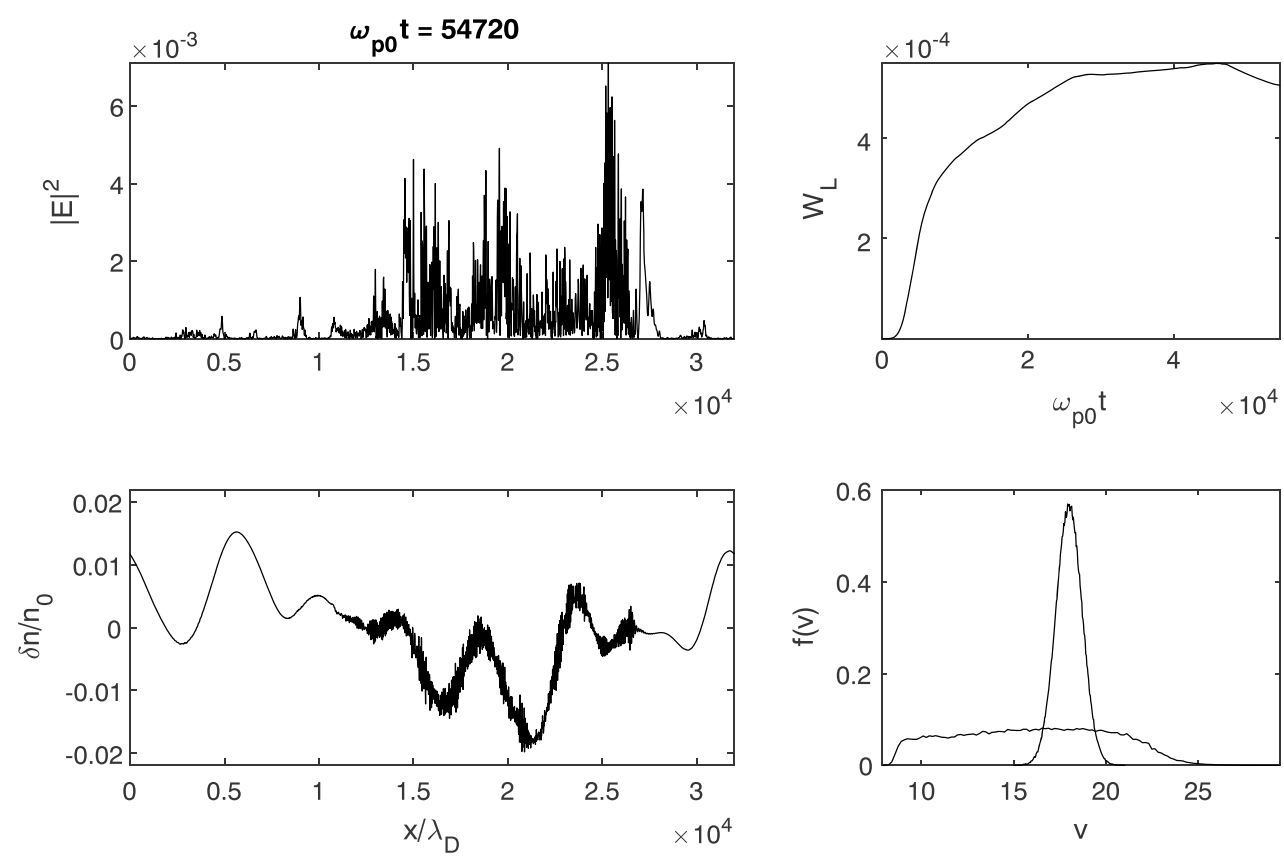

Figure 7. (left column) Space profiles of the Langmuir energy density $|E|^{2}$ and of the density fluctuations $\delta n / n_{0}$, at time $\omega_{p 0} t=54,720$. (right column) Time evolution of the Langmuir wave energy density $W_{L}$ and of the beam velocity distributions $f(v)$ at $\omega_{p 0} t=0$ and $\omega_{p 0} t=54,720$. The average level of density fluctuations is $\Delta n=0.01$. The ratio of the beam to the plasma density is $n_{b} / n_{0}=0.00002$.

In the presence of background long-wave density fluctuations ( $\Delta n \gg 0.001$ ), the electric fields' amplitudes' profiles reveal the formation of peaks of localized wave energy due to focusing processes, as one can see in Figure 7 for a simulation with $\Delta n \simeq 0.01$. This property is maintained until the development of the decay instability leads to a significant broadening of the spectrum.

As several narrow wave packets dominate the energy density profile, the PDF exhibits two smooth maxima and a plateaued area extending within the range $10^{-6} \lesssim|E|^{2} \lesssim 10^{-3}$ (Figure 8). However, at low amplitudes, i.e., $|E|^{2} \lesssim 10^{-6}$, the scaling is similar to the above case $(\Delta n \leq 0.001)$, i.e., $P\left(\log _{10}|E|^{2}\right) \sim \alpha_{1}\left(\log _{10}|E|^{2}\right)$, with $\alpha_{1} \simeq 2.1$. We will see below that in all the cases studied the probability distributions present at small amplitudes $|E|$ the same universal scaling, with $\alpha_{1}$ not deviating significantly from 2 . When looking at a more advanced stage of the Langmuir turbulence, one observes that the structure of the PDF with two smooth maxima and a plateaued area disappears, corresponding actually to transient conditions that will no more be considered hereafter.
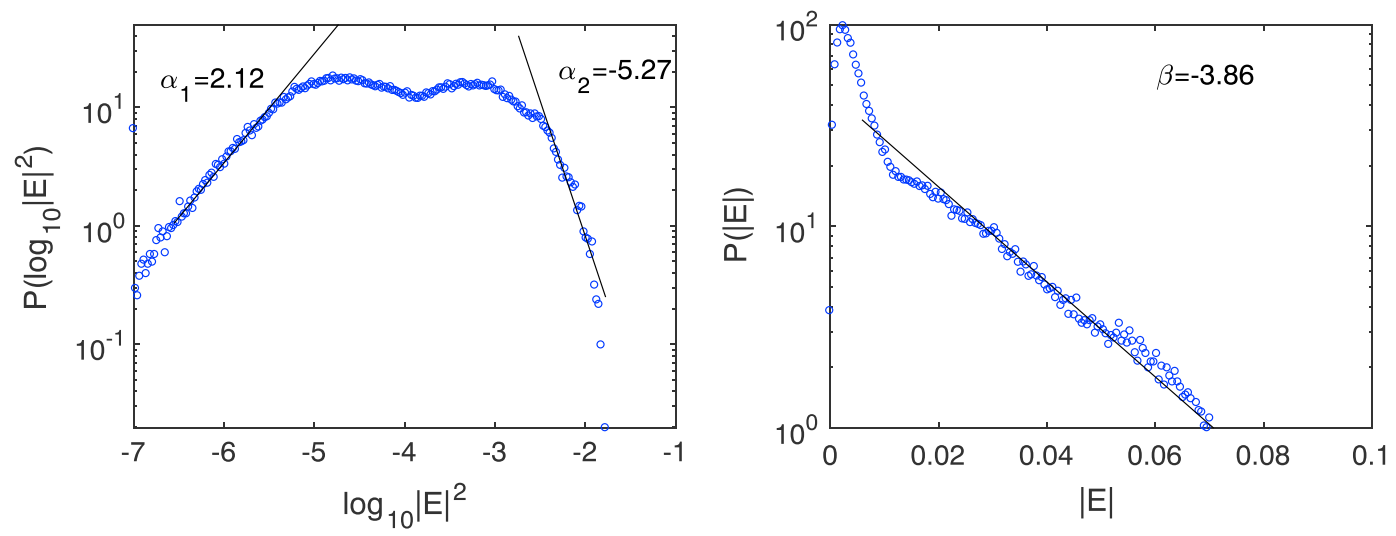

Figure 8. Averaged PDFs of the electric fields' amplitudes obtained for the simulation presented in Figure 7, in the normalized time interval $\Delta T=[35,000-45,000]$. (left) Distribution of $\log _{10}|E|^{2}$, with linear fits' coefficients $\alpha_{1} \simeq 2.1$ and $\alpha_{2} \simeq-5.3$. (right) Distribution of $|E|$, in logarithmic scale, with the linear fit's coefficient $\beta \simeq-3.8$. 


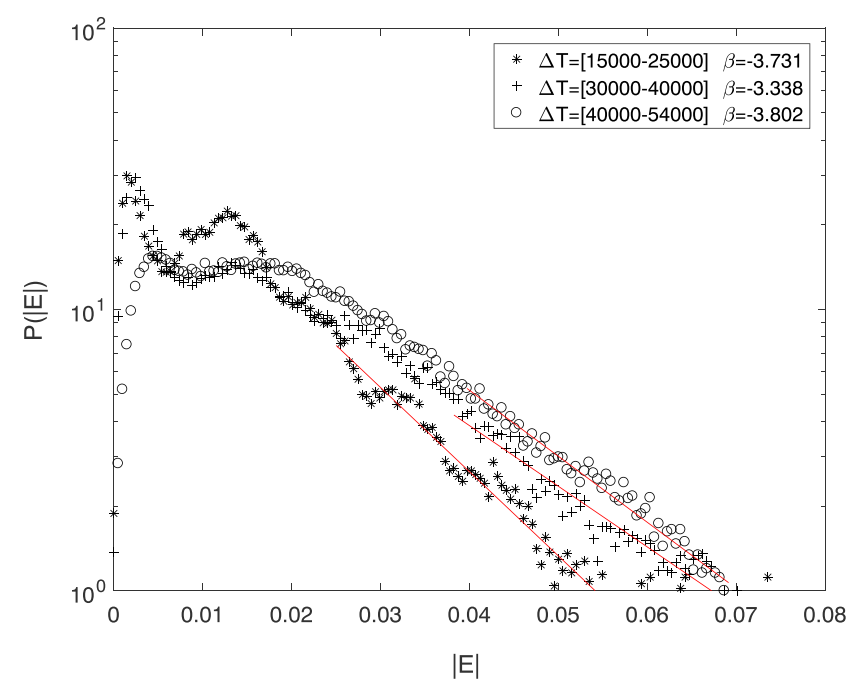

Figure 9. Probability distributions $P(|E|)$ of the electric fields' amplitudes $|E|$, in logarithmic scale, obtained for three different time intervals $\Delta T$ inside the spatial region $\Delta x / \lambda_{D}=[9000,25,000]$; the straight lines represent three linear fits. The time intervals as well as the corresponding interpolation coefficients $\beta$ are indicated in the insert. The parameters are those of the simulation presented in Figure 7.

In the region of large amplitudes $|E|$, no universal scaling of the form $P\left(\log _{10}|E|^{2}\right) \sim \alpha_{2} \log _{10}|E|^{2}$ has been found. In this case, it is more appropriate to consider $P(|E|)$, whose approximation at large amplitudes is usually close to the exponential interpolation $P(|E|) \sim \exp \left(\beta|E| /|E|_{m}\right)$. These distributions evolve in time according to the wave spectrum and the total energy density $W_{L}$. Figure 9 shows the PDFs obtained for the same simulation as in Figure 8, but within three different time intervals $\Delta T$. One can see that the slope of the function $P(|E|)$ (which is represented in logarithmic scale by the parameter $\beta$ ) does not depend substantially on $\Delta T$ as $-3.8 \lesssim \beta \lesssim-3.3$. At the same time, the development of wave decay instabilities results in a decrease of the largest bursts' amplitudes and in the appearance of other peaks in the spatial profile of $|E|^{2}$. As a result, the profiles of the PDFs become smoother with time.

However, if we consider the simulation of a fairly large system with a large number of wave packets, the smoothing of the PDF can be obtained prior to the development of the decay instability. This can be illustrated in Figure 10 by the calculation of the PDFs using four different time intervals $\Delta T$, for a case with $\Delta n \simeq 0.01$. Only the results obtained for the first interval $\Delta T=[6000-8000]$ are substantially different from the others (strongly scattered points), while those provided by the three other $\Delta T$ show the same scaling with rather

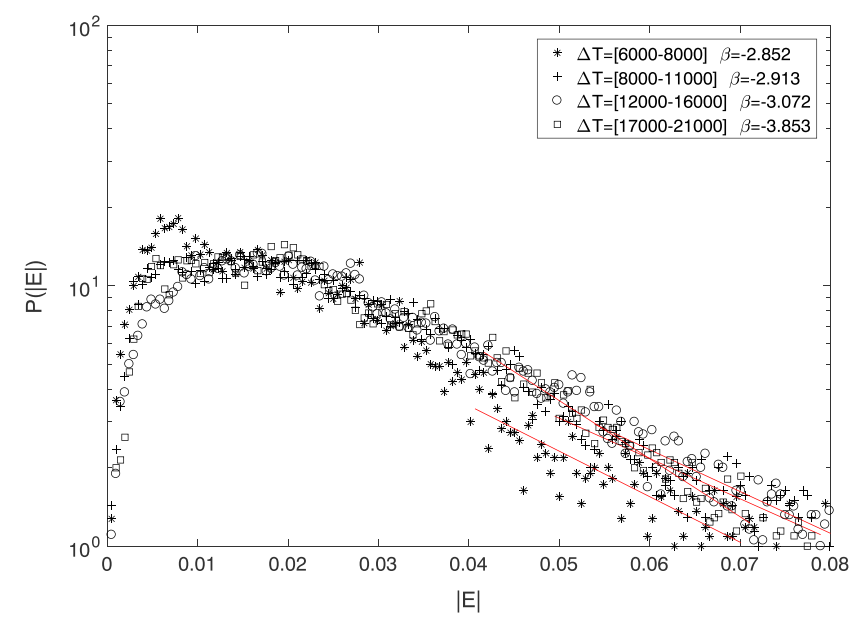

Figure 10. Probability distributions of the electric fields' amplitudes $|E|$, in logarithmic scale, obtained for four different time intervals $\Delta T$, as indicated in the insert with the corresponding interpolation parameters $\beta$; the straight lines represent four linear fits. The average level of fluctuations is $\Delta n=0.01$. 


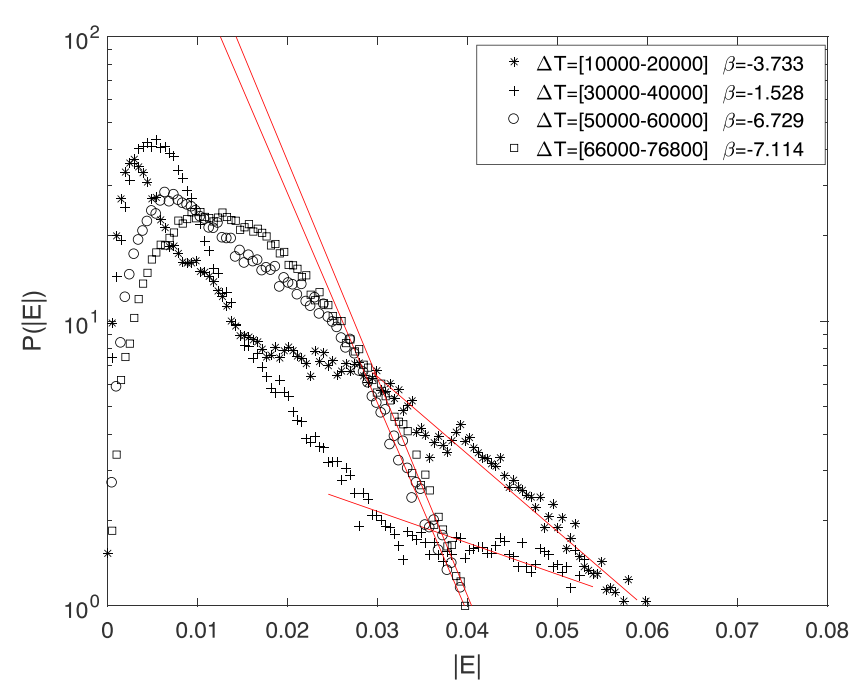

Figure 11. Probability distributions of the electric fields' amplitudes $|E|$, in logarithmic scale, obtained for four different time intervals $\Delta T$, as indicated in the insert with the corresponding interpolation parameters $\beta$; the straight lines represent four linear fits. The average level of fluctuations is $\Delta n=0.01$.

close characteristic parameters, i.e., $-3.8 \lesssim \beta \lesssim-3.3$. Despite the fact that the decay instability becomes visible only after $\omega_{p 0} t=11,000$, the PDF obtained using the interval $\Delta T=[8000-11,000]$ is not very different from that calculated within $\Delta T=[12,000-16,000]$, as $\beta \simeq-3$ for both cases.

When considering a longer-term evolution of the Langmuir turbulence and studying the corresponding changes in the PDFs $P(|E|)$, one observes a clear trend toward a universal asymptotic approximation, not only for the small amplitudes $|E|$ but also for the large ones. Figure 11 shows the PDFs calculated for a simulation with $\Delta n \simeq 0.01$ within different time intervals $\Delta T$. Note that they are very close for $\Delta T=[50,000-60,000]$ and $\Delta T=[66,000-76,800]$. At the same time, an exponential approximation is good only for the last end of the amplitudes' distributions and is less accurate in the range of the mean amplitudes. Furthermore, one can see that the probability of encountering very high amplitudes decreases with time (for the same level of turbulence), which means, in particular, that the electric energy density is distributed in more peaks of smaller amplitudes. Note finally that despite the presence of background density fluctuations, the asymptotic form of the PDFs is similar to that shown in Figure 6 for the case $\Delta n \simeq 0$.

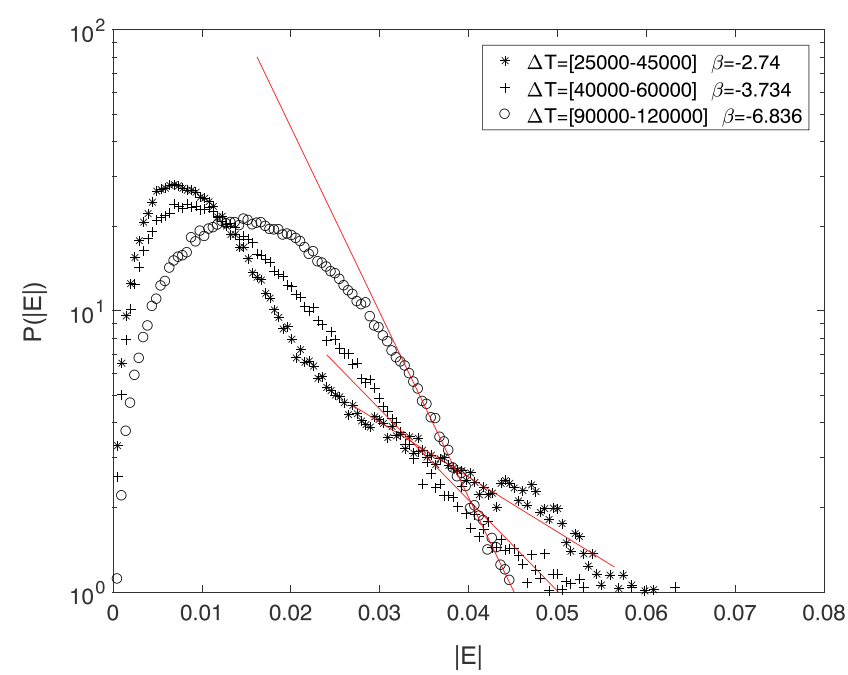

Figure 12. Probability distributions of the electric fields' amplitudes $|E|$, in logarithmic scale, obtained for three different time intervals $\Delta T$, as indicated in the insert with the corresponding interpolation parameters $\beta$; the straight lines represent three linear fits. The average level of fluctuations is $\Delta n=0.02$. 

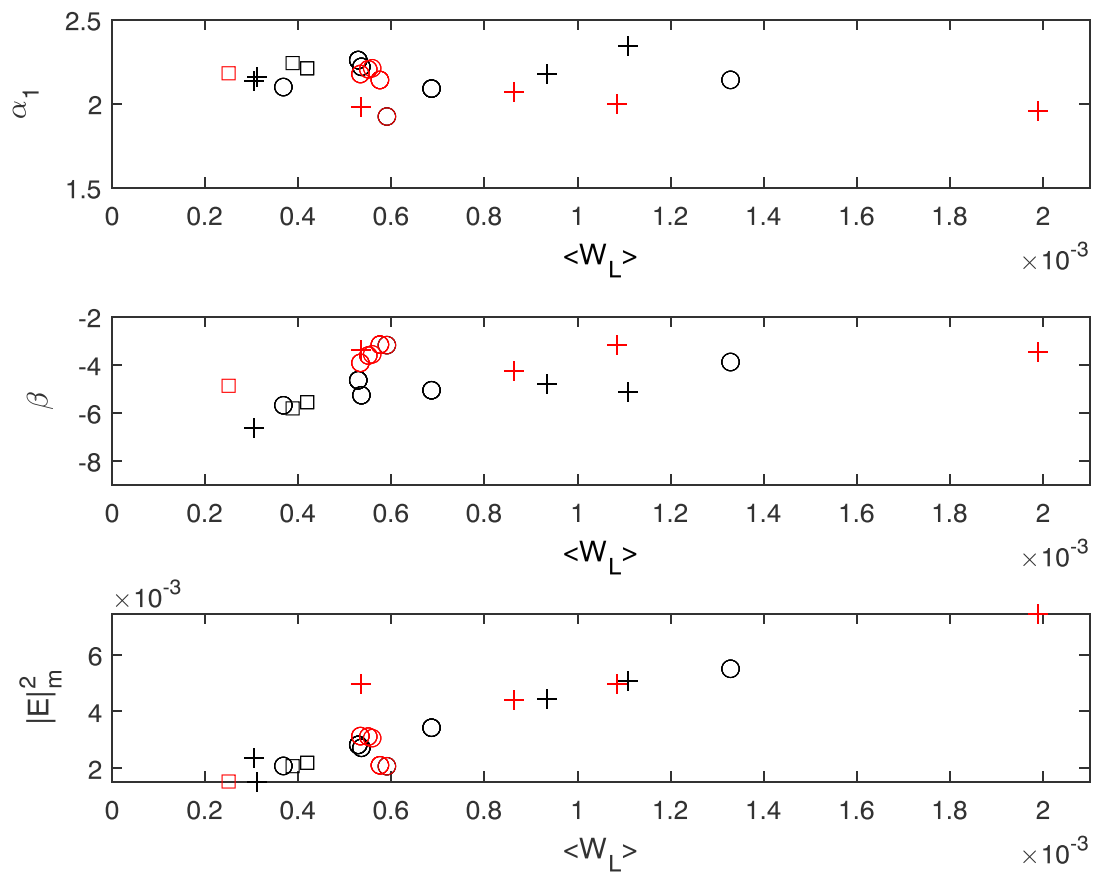

Figure 13. Characteristic parameters (top) $\alpha_{1}$, (middle) $\beta$, and (bottom) $|E|_{m}^{2}$ of the probability distributions of the Langmuir electric fields, obtained for simulations with different average levels of density fluctuations: $\Delta n \simeq 0.01$ (crosses), $\Delta n \simeq 0.02$ (circles), $\Delta n \simeq 0.03$ (squares). The red (respectively, black) markers indicate that the decay instability does not play (respectively, plays) an essential role in the corresponding simulations.

The same trend in the time evolution of the PDFs is visible in another example with $\Delta n \simeq 0.02$ (Figure 12). At large amplitudes the PDFs present an asymptotic behavior; i.e., $P(|E|) \sim \exp \left(\beta|E| /|E|_{m}\right)$, with $\beta \simeq-3.5$ until the time when the decay instability leads to significant changes in the wave spectra. Then, at further times, $\beta$ decreases down to the range $-8 \lesssim \beta \lesssim-5$, whereas the PDFs become more and more close to a universal asymptotic form similar to Figure 6 (right).

All the examples of the PDFs of the electric fields' amplitudes $|E|$ calculated by the simulations based on the dynamical model correspond to limited sets of initial conditions (density fluctuations, etc.). Nevertheless, it is useful to provide a summary of the PDFs' features for different electron beam velocities and densities, density fluctuations' profiles with $0<\Delta n \lesssim 0.03$ and time intervals $\Delta T$. Thus, we present in Figure 13 the interpolation parameters $\alpha_{1}$ and $\beta$ as well as the statistical maximum $|E|_{m}$ of the electric fields' amplitudes $|E|$ as a function of the wave energy $\left\langle W_{L}\right\rangle$ averaged on the time intervals in which the PDFs are calculated. The scattering of these parameters may be the result of the lack of statistics due to the small size of the modeling system and to the fact that in some cases we analyzed transition states too close to the initial stage of the beam instability. Note that the parameter $\alpha_{2}$ is not shown here as its values are too much scattered.

Except cases with insufficiently accurate approximations and not well-established PDFs, one can draw the following conclusions. First, in the region of small electric fields' amplitudes, the scaling of the PDFs is determined by $P\left(\log _{10}|E|^{2}\right) \sim \alpha_{1} \log _{10}|E|^{2}$, with $2 \lesssim \alpha_{1} \lesssim 2.3$; this value does not seemingly depend on the level of density fluctuations $\Delta n$ and on the wave energy $\left\langle W_{L}\right\rangle$. Second, when decay instabilities are weak or even absent, the scaling implemented with the exponential asymptotic behavior $P(|E|) \sim \exp \left(-\beta|E| /|E|_{m}\right)$ operates in a wide range of fields' amplitudes $|E| \leq|E|_{m}$; moreover, the parameter $-6 \lesssim \beta \lesssim-3$ decreases when approaching the universal probability distribution realized when the decay processes are sufficiently strong. Third, the value of the statistical maximum $|E|_{m}$ of the electric fields' amplitudes correlates well with the average energy of the Langmuir waves, i.e., $|E|_{m}^{2} \propto\left\langle W_{L}\right\rangle$. Note also that, in some cases, the corresponding large-amplitude portions of the PDFs can be described by $P\left(\log _{10}|E|\right) \sim \alpha_{2} \log _{10}|E|$, with $-8 \lesssim \alpha_{2} \lesssim-2$.

Note that these conclusions could be made more accurate if using a larger statistical ensemble, which should include longer intervals of space and a greater variety of initial density fluctuations' profiles. The obvious 
limitations of the numerical resources make this task difficult. However, results provided by the simulations based on the dynamical model are close to those obtained above using the probabilistic model.

\section{Conclusion}

We have performed a statistical analysis of the Langmuir waves' fields' amplitudes using numerical simulations based on two different theoretical models, i.e., a probabilistic one and a dynamical one. In both cases the probability distributions of the fields' amplitudes are presented in the whole similar features.

The probabilistic model of beam-plasma interaction includes probability distributions of density fluctuations. To carry out the analysis of the statistical distributions of the wave fields' amplitudes, we created synthetic data sets using selected density profiles with power spectra statistically similar to those observed in the solar wind. The WKB approximation was used to reconstruct the spatial distributions of the electric fields on scales smaller than the typical scale of the beam relaxation. In order to take into account the influence of the density irregularities, we calculated the spatial distributions of the wave vectors from those of the density fluctuations. Slow temporal changes in $E$ were taken from the numerical simulations based on the probabilistic model.

The analysis of the field data containing clumpy structures shows that the cores of the PDFs of the logarithms of the fields' amplitudes correspond to Pearson type IV distributions. This result is consistent with that obtained by Krasnoselskikh et al. [2007]; however, it disagrees with the normal distribution predicted by the SGT [Robinson et al., 1993] and obtained by the analysis of the experimental observations on board satellites [Cairns and Robinson, 1999; Píša et al., 2015; Briand et al., 2016]. The reason for the deviations of the distributions from the normal ones could consist in the fact that the effective number of regions where the clumps are observed is not very large and, as a consequence, the central limit theorem fails to be true. Regardless of the clumps' presence, the distributions of the reconstructed wave fields correspond to three main types of Pearson distributions, namely, types I, IV, and VI. It is worth noting that a similar result was obtained in Vidojević et al. [2011] by using the Wind spacecraft data. Also, our analysis shows that the high-amplitude parts of the distributions follow power law or exponential decay behaviors, depending on the type of the cores' distributions. Note that power law tails with exponent 1 were found for the distributions of the Langmuir waves observed within the Earth's electron foreshock and in the vicinity of other planetary shocks [Bale et al., 1997; Cairns and Robinson, 1999; Boshuizen et al., 2004; Píša et al., 2015].

The probability distributions of the fields' amplitudes calculated using the numerical simulations based on the dynamical model are in the whole consistent with those provided by the probabilistic model. Moreover, these simulations lead to a series of additional results. First, in the small fields' amplitudes' parts of the PDFs (i.e., in the linear stage of the system's evolution), a universal scaling parameter $\alpha_{1}$ of the distributions is found, with a value not depending on the average levels of the density fluctuations and of the Langmuir turbulence. Second, probability distributions are obtained in the presence of wave decay processes, which are not taken into account in the probabilistic model; when these processes are weak, the PDFs show at large fields' amplitudes an exponential asymptotic behavior within a wide range of $|E| \leq|E|_{m}$; during the time evolution, one observes that the corresponding scaling parameter $\beta$ decreases until a universal probability distribution is reached, which is realized when the wave decay processes are sufficiently strong. This distribution is analogous to that obtained for a quasi-homogeneous plasma. Note also that such exponential type of distribution is a specific signature of transition states in the Langmuir turbulence. Third, the square of the statistical field amplitude maximum $|E|_{m}$ is found to be proportional to the average energy of the Langmuir waves.

Both models exhibit the formation of spikes of Langmuir energy density with magnitudes several times above the mean. Since the probabilistic model is operated by the averaged waves' growth rate, the gain of wave energy takes place on spatial scales much larger than the scale of a single density irregularity, and the model cannot describe a significant amplification of $|E|^{2}$ on the characteristic scales of the clumps. Therefore, one can conclude that the observed clumpy structures might appear due to effects related to the propagation of the Langmuir waves in the inhomogeneous plasma rather than to the oscillations of the waves' growth rates.

\section{Appendix A: Probabilistic Model of the Beam-Plasma Interaction}

The model allows to estimate statistically the averaged rate of energy exchange between waves and particles that interact at Cherenkov resonance conditions. Using a procedure of averaging, it takes into account 
the fact that the phase velocity of a Langmuir wave undergoes variations as the wave propagates within the fluctuating plasma. This implies that the wave can resonantly interact with different groups of electrons when passing through regions of different plasma density.

A justification for the averaging procedure can be found in the naturally occurring scales' separation. The development of the bump-on-tail instability is characterized by two intrinsic times: a time $t_{\mathrm{nl}}$ related to the wave-particle interaction (typically of the order of several periods of oscillations of the resonant electrons) and a time $t_{\mathrm{ql}}$ during which significant changes in the electron velocity distribution occur (inversely related to the wave growth rate). During $t_{\mathrm{nl}}$ the velocities of the resonant electrons undergo relatively small variations, and substantial changes in the velocity distribution occur on timescales $t_{\mathrm{ql}} \gg t_{\mathrm{n} \mid}$. As a result, the electrons can be accelerated or decelerated depending on the waves' phases. As a consequence, the motion of a selected group of electrons resemble a random walk within the velocity space; averaged on the scale $t_{\mathrm{q}}$, this motion manifests itself as a slow diffusion of the electron velocity distribution function.

The situation is much more complicated in a plasma with a fluctuating density. One of the effects related to the density fluctuations is the variation of the wave phase velocity. As a result, during the time interval $t_{\mathrm{ql}}$, the wave can resonantly interact with groups of electrons having different velocities. To deal with this problem, it was suggested to introduce a probability distribution function $P_{\omega_{i}}(V)$ that describes the variations of the waves' phase velocities: $P_{\omega_{i}}(V) \mathrm{d} V$ is the probability to find a wave of frequency $\omega_{i}$ with a phase velocity $V$ at any particular time in the interval $t_{\mathrm{nl}}$. It was shown in Voshchepynets et al. [2015] that, by making use of $P_{\omega_{i}}(V)$, the beam relaxation can be described self-consistently by a system of equations similar to those of the quasi-linear theory for homogeneous plasmas, but with the canonical electron velocity diffusion coefficient and the waves' growth rate replaced by the corresponding ones averaged in the velocity space. In the general case, for beam electrons with a velocity distribution function $f(v, t)$ and a density $n_{b}$ interacting with waves of frequencies $\omega_{i}$ and wave energy densities $W_{i}$, the system's equations are the following:

$$
\begin{gathered}
\frac{\partial f(v, t)}{\partial t}=\frac{2 \pi^{2} e^{2}}{m_{e}} \frac{\partial}{\partial v} \sum_{i} \frac{v W_{i} P_{\omega_{i}}(v)}{\omega_{i}} \frac{\partial f(v, t)}{\partial v} \\
\frac{\mathrm{d} W_{i}}{\mathrm{~d} t}=\pi \omega_{p 0} W_{i} \frac{n_{b}}{n_{0}} \int_{0}^{\infty} V^{2} P_{\omega_{i}}(V)\left(\frac{\partial}{\partial v} f(v, t)\right)_{v=v} \mathrm{~d} V
\end{gathered}
$$

It is worth noting that the system conserves the total energy carried by the waves and the particles, which is suitable for numerical simulations of the beam-plasma interaction. However, we should admit that the only effect included in the model is the resonance broadening due to the variations of the waves' phase velocities in the fluctuating plasma. Thus, results obtained using the probabilistic model should be considered as a limiting case, when all other processes involved in the development of Langmuir turbulence (such as wave-wave interactions) can be neglected.

The probability distributions $P_{\omega_{i}}(V)$ play a key role in the model. They can be uniquely determined from the probability distributions of the density fluctuations, by making use of the nonlinear dispersion relation for Langmuir waves. These distributions can be taken from models allowing calculations to be performed in a simpler manner, or they can correspond to more realistic observations of density fluctuations in the solar wind. Voshchepynets and Krasnoselskikh [2015] have shown that the Pearson type II distributions might be considered as the best fit for the distribution function of density fluctuations deduced from the two-knee power law spectrum observed by satellites [Celnikier et al., 1987]. In the following study we use the distribution $P_{\omega_{i}}(V)$ obtained from a Pearson type II distribution.

In the absence of fluctuations, the phase velocity of a Langmuir wave generated by a bump-on-tail instability remains constant during the wave propagation. In this case, $P_{\omega_{i}}(V)$ tends to a Dirac function, and the system of equations (A1) and (A2) takes the same form as the corresponding system used in the quasi-linear approach for a homogeneous plasma. The presence of density fluctuations leads to the variations of the waves' phase velocities and results in an asymmetrical broadening of $P_{\omega_{i}}(V)$ [Voshchepynets et al., 2015]. Increasing the amplitudes of the density fluctuations leads to a widening of the velocity domain where the wave can resonantly interact with electrons. The resonance broadening can cause the self-acceleration of the beam electrons via the generation and the absorption of the waves by groups of electrons with different velocities. 

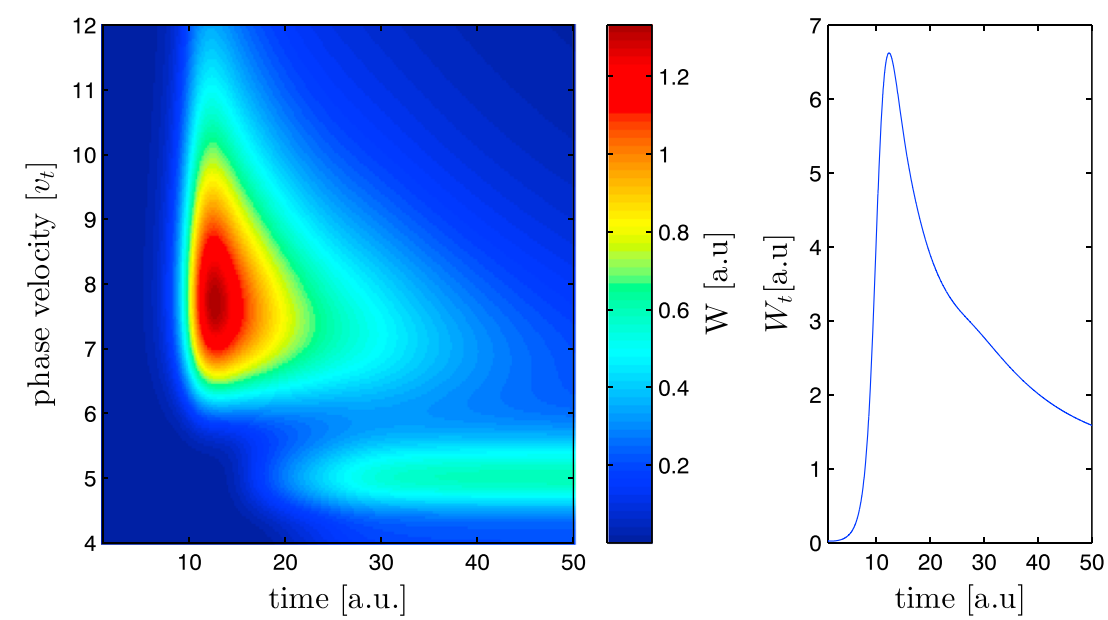

Figure A1. Evolution of the wave spectral energy densities $W(V)$ as (left) a function of time and phase velocity $V$ and (right) variation with time of the total wave energy density $W_{t}$. Wave energy density is normalized to $2 n_{b} m_{e} v_{t}^{2}$, while $W(V)$ is normalized to $2 n_{b} m v_{t}$ and time to $\left(2 \pi \omega_{p 0}\right)^{-1}\left(n_{b} / n_{0}\right)$. The average level of the density fluctuations is $\Delta n=0.02$.

The system of equations is solved by applying a Leapfrog method and the Simpson's rule is used to perform the integration in (A2). Parameters of the driven beam and the ambient plasma are chosen to be close to those observed by satellites in the solar wind [Ergun et al., 1998], namely, background plasma density: $n_{0} \sim 5 \cdot 10^{6} \mathrm{~m}^{-3}$; averaged plasma frequency, $\omega_{p 0} / 2 \pi \sim 20 \mathrm{kHz}$; beam density, $n_{b} / n_{0} \sim 10^{-5}$; and Debye length, $\lambda_{D} \sim 15 \mathrm{~m}$. The initial beam velocity distribution $f(v)$ is a Gaussian, with a drift velocity $v_{b}=14 v_{t}$ ( $v_{t}$ is thermal velocity of the background plasma) and a thermal velocity $\Delta v_{b}=0.08 v_{b}$. A set of 2000 Langmuir waves is used, with phase velocities $v_{i}$ uniformly distributed in the range $\left[3 v_{t}, 40 v_{t}\right]$. The initial energy density of each wave is chosen as $W_{i}=5 \cdot 10^{-7} \cdot E_{b}$, where $E_{b}$ is the initial energy density of the beam.

We performed simulations with different average levels $\Delta n$ of density fluctuations, varying from 0.01 to 0.04 . The temporal evolution of the wave spectral energy density $W(V)=\sum_{i} W_{i}\left(V_{i}\right) P_{\omega_{i}}(V)$ (left) and of the total wave energy density $W_{t}$ (right) obtained by simulations with $\Delta n=0.02$ are shown in the Figure A1. For the sake of simplicity, the results are provided in arbitrary units.

At the initial stage, the Langmuir turbulence develops in a wide range of phase space. However, due to the resonance broadening, the waves with large phase velocities start to transfer energy to the electrons more effectively than they can gain it from them. For instance, the intense peak in the wave spectral energy density that appears in the range of phase velocities from $7 v_{t}$ to $9 v_{t}$ at the time $t \gtrsim 10$ au has completely vanished at $t>30 \mathrm{au}$. Another interesting result is the development of a second peak around $5 v_{t}$, starting approximately at $t \simeq 20$ au. This group of waves is generated by the relaxation front of the electron velocity distribution when reaching the thermal range. Contrary to the first peak, the intensity of the second one remains constant after it has reached its maximum. As it was shown in Voshchepynets et al. [2015], the density fluctuations result in a sufficient broadening of the $P_{\omega_{i}}(V)$ only for those waves that satisfy the condition $\left(v_{t} / V_{i}\right)^{2}<\Delta n$. Thus, the fluctuations with $\Delta n=0.02$ cannot cause a notable effect on the development of the second group of waves.

The temporal evolution of the total wave energy density, shown in Figure A1 (right), can be used to illustrate the peculiarities of the beam-plasma interaction in a plasma with density fluctuations. First, the efficiency of the beam-plasma instability is reduced in comparison to the case of a homogeneous plasma. The quasi-linear theory [Vedenov, 1967] provides an estimation of the maximum of wave energy density that can be reached during the relaxation process, i.e., $W^{\mathrm{QL}}=n_{b} m v_{b}^{2} / 3$. For the beam with $v_{b}=14 v_{t}$ used in our simulation, the QL theory provides that $W_{t}^{\mathrm{QL}} \sim 32 \mathrm{au}$. The maximum of $W_{t}$ observed in a simulation with $\Delta n=0.02$ is around $7 \mathrm{au}$, which is only slightly above $20 \%$ of the energy that waves could gain from the beam in a homogenous plasma. Second, the density fluctuations cause the reabsorption of part of the wave energy by the electron beam. After the wave energy density reaches its maximum, it starts to decrease whereas the energy of the energetic electrons increase. An energy transfer from one group of electrons to another, via the generation and the absorption of Langmuir waves, can be very efficient in a plasma with high levels of density fluctuations, leading to the self-acceleration of the beam particles. 


\section{Appendix B: Dynamical Model of Langmuir Turbulence Excited by Beams}

The 1-D theoretical model describes the self-consistent interaction of Langmuir waves with electron beams in plasmas with randomly varying density inhomogeneities. The dynamics of Langmuir and ion sound waves is calculated using the two Zakharov's equations [Zakharov, 1972], where a source term is added to model the beam. As shown in a previous paper [Krafft et al., 2013], these equations can be written as

$$
\begin{gathered}
i \frac{\partial E}{\partial t}+\frac{3 \lambda_{D}^{2}}{2} \omega_{p 0} \frac{\partial^{2} E}{\partial x^{2}}-\omega_{p 0} \frac{\delta n}{2 n_{0}} E=4 \pi i e n_{b} \sum_{k} \frac{\omega_{p 0}}{k} \frac{1}{N} \sum_{p} e^{i \omega_{p 0} t-i k x_{p}} e^{i k x}, \\
\left(\frac{\partial^{2}}{\partial t^{2}}-c_{s}^{2} \frac{\partial^{2}}{\partial x^{2}}\right) \frac{\delta n}{n_{0}}=\frac{\partial^{2}}{\partial x^{2}} \frac{|E|^{2}}{16 \pi m_{i} n_{0}},
\end{gathered}
$$

where $x$ is the coordinate along the ambient magnetic field $\mathbf{B}_{0} ; \omega_{p 0}$ and $\lambda_{D}$ are the electron plasma frequency and Debye length; $k$ is the wave number of the Langmuir wave of frequency $\omega_{k} \simeq \omega_{p 0}+3 \omega_{p 0} k^{2} \lambda_{D}^{2} / 2$; $\mathcal{E}=\Re\left(E(x, t) e^{-i \omega_{p 0} t}\right)$ is the electric field, and $E(x, t)$ is its slowly varying envelope; $\delta n$ is the low-frequency density perturbation; $m_{i}$ and $m_{e}$ are the ion and electron masses; $-e<0$ is the electron charge; $n_{b}$ is the beam density; $T_{i}$ and $T_{e}$ are the ion and electron temperatures, which are supposed to satisfy the condition $T_{i} \ll T_{e} ; c_{s}=\sqrt{\left(T_{e}+3 T_{i}\right) / m_{i}}$ is the ion acoustic velocity; $x_{p}$ is the position of the particle $p ; N$ is the number of macroparticles, i.e., the number of resonant electrons.

The model divides the total particle distribution in two groups: (i) the background plasma whose particles interact nonresonantly with the waves, and (ii) the beam particles which exchange resonantly energy and momentum with the waves [O'Neil et al., 1971; Volokitin and Krafft, 2004; Krafft et al., 2005; Krafft and Volokitin, 2006; Zaslavsky et al., 2006, 2007; Krafft et al., 2010, 2013]. The first group of electrons supports the wave dispersion, and its dynamics is modeled using the dielectric constant in the frame of a linear approach (see also the beam source term in (B1)). On another hand, the resonant electrons of velocity $v$ exchange momentum and energy with the waves at the Landau resonances $\omega_{k} \simeq \omega_{p 0} \simeq k v$. Their dynamics is calculated by solving the Newton equations. Such approach leads to a drastic reduction of the number of macroparticles required in the calculations, giving the possibility to study the microscopic beam dynamics and the Langmuir turbulence over long periods of time. Nevertheless, it is required that the resonant particles' density $n_{b}$ be much less than the ambient plasma density; i.e., $n_{b} \ll n_{0}$.

The Newton equations for the $N$ particles $p$ have to be added to equations (B1) and (B2)

$$
m_{e} \frac{\mathrm{d} v_{p}}{\mathrm{~d} t}=-e \mathcal{E}\left(x_{p}, t\right)=-e \Re\left(\sum_{k} E_{k} e^{i k x_{p}-i \omega_{k} t}\right), \quad \frac{\mathrm{d} x_{p}}{\mathrm{~d} t}=v_{p},
$$

where $v_{p}$ is the velocity of the electron $p_{;} E_{k}$ is the Fourier component of $E$

$$
E_{k}(t)=\int_{0}^{L} E(x, t) e^{-i k x} \frac{\mathrm{d} x}{L},
$$

where $L=N / n_{b}$ is the size of the system. Rewriting equation (B1) in the $k$ space, one obtains that

$$
i\left(\frac{\partial}{\partial t}-\gamma_{k}^{(e)}\right) E_{k} \simeq \frac{3}{2} \omega_{p 0} k^{2} \lambda_{D}^{2} E_{k}+\frac{\omega_{p 0}}{2}(\rho E)_{k}+i \frac{4 \pi e \omega_{p 0} n_{b}}{k} J_{k},
$$

where $\rho=\delta n / n_{0}$ and $J_{k}=(1 / N) \sum_{p} \exp \left(i \omega_{p 0} t-i k x_{p}\right)$. A kinetic damping factor $\gamma_{k}^{(e)}=-\Im \varepsilon_{k}^{(e)} /\left(\partial \Re \varepsilon_{k}^{(e)} / \partial \omega_{k}\right)$ (where the subscript (e) refers to electrons) is added eventually in (B5) in order to take into account the damping of the plasma waves when interacting with thermal particles or with nonthermal electrons of the background plasma distribution, as, for example, non-Maxwellian tails.

The Fourier transforms of equation (B2) and of the plasma continuity equation lead to the following expressions (ion damping is not included):

$$
\begin{gathered}
\frac{\partial}{\partial t} \rho_{k} \simeq i k c_{s} u_{k}, \\
\frac{\partial u_{k}}{\partial t} \simeq i k c_{s}\left(\rho_{k}+\frac{\left(|E|^{2}\right)_{k}}{16 \pi m_{i} n_{0} c_{s}^{2}}\right),
\end{gathered}
$$

where $v_{i}$ and $u=v_{i} / c_{s}$ are the ion velocity and its normalized value. Equations (B5)-(B7) together with equation (B3) form the complete set of equations of the dynamical model. 
Acknowledgments

V.K. acknowledges the financial support of the Centre National d'Etudes Spatiales (CNES) through the grants "Accompagnement scientifique de Solar Orbiter" and "Invited scientist STEREO/WAVES". A.V. acknowledges financial support of CNES through the grant "Accompagnement scientifique de Solar Orbiter." This work was granted access to the HPC resources of IDRIS under the allocation 2013-i2013057017 made by GENCI. This work has been done within the LABEX Plas@par project and received financial state aid managed by the Agence Nationale de la Recherche, as part of the program "Investissements d'avenir" under the reference ANR-11-IDEX-0004-02. C.K. acknowledges the "Programme National Soleil Terre" (PNST) and the Centre National d'Etudes Spatiales (CNES, France).

\section{References}

Austin, D. R., M. J. Hole, P. A. Robinson, I. H. Cairns, and R. Dallaqua (2007), Laboratory evidence for stochastic plasma-wave growth, Phys. Rev. Lett., 99(20), 205004, doi:10.1103/PhysRevLett.99.205004.

Bale, S. D., D. Burgess, P. J. Kellogg, K. Goetz, and S. J. Monson (1997), On the amplitude of intense Langmuir waves in the terrestrial electron foreshock, J. Geophys. Res., 102, 11,281-11,286, doi:10.1029/97JA00938.

Bale, S. D., P. J. Kellogg, D. E. Larsen, R. P. Lin, K. Goetz, and R. P. Lepping (1998), Bipolar electrostatic structures in the shock transition region: Evidence of electron phase space holes, Geophys. Res. Lett., 25, 2929-2932, doi:10.1029/98GL02111.

Bendat, J. S., and A. G. Piersol (1980), Engineering Applications of Correlation and Spectral Analysis, 315 pp., Wiley-Interscience, New York.

Boshuizen, C. R., I. H. Cairns, and P. A. Robinson (2004), Electric field distributions for Langmuir waves in planetary foreshocks, J. Geophys. Res., 109, A08101, doi:10.1029/2004JA010408.

Briand, C., P. Henri, V. Génot, N. Lormant, N. Dufourg, B. Cecconi, Q. N. Nguyen, and K. Goetz (2016), STEREO database of interplanetary Langmuir electric waveforms, J. Geophys. Res. Space Physics, 121, 1062-1070, doi:10.1002/2015JA022036.

Cairns, I. H., and J. D. Menietti (2001), Stochastic growth of waves over Earth's polar cap, J. Geophys. Res., 106, 29,515-29,530, doi:10.1029/2000JA000422.

Cairns, I. H., and P. A. Robinson (1997), First test of stochastic growth theory for Langmuir waves in Earth's foreshock, Geophys. Res. Lett., 24, 369-372, doi:10.1029/97GL00084.

Cairns, I. H., and P. A. Robinson (1999), Strong evidence for stochastic growth of Langmuir-like waves in Earth's foreshock, Phys. Rev. Lett., 82, 3066-3069, doi:10.1103/PhysRevLett.82.3066.

Celnikier, L. M., L. Muschietti, and M. V. Goldman (1987), Aspects of interplanetary plasma turbulence, Astron. Astrophys., 181, $138-154$.

Chen, C. H. K., C. S. Salem, J. W. Bonnell, and F. S. Mozer (2012), Density fluctuation spectrum of solar wind turbulence between ion and electron scales, Phys. Rev. Lett., 109(3), 035001, doi:10.1103/PhysRevLett.109.035001.

Ergun, R. E., et al. (1998), Wind spacecraft observations of solar impulsive electron events associated with solar type III radio bursts, Astrophys. J., 503, 435-445, doi:10.1086/305954.

Ginzburg, V. L., and V. V. Zhelezniakov (1958), On the possible mechanisms of sporadic solar radio emission (radiation in an isotropic plasma), Sov. Astron., 2, 653

Kellogg, P. J., and T. S. Horbury (2005), Rapid density fluctuations in the solar wind, Ann. Geophys., 23, 3765-3773, doi:10.5194/angeo-23-3765-2005.

Kellogg, P. J., K. Goetz, S. J. Monson, and S. D. Bale (1999), Langmuir waves in a fluctuating solar wind, J. Geophys. Res., 104, 17,069-17,078, doi:10.1029/1999JA900163.

Krafft, C., and A. Volokitin (2006), Stabilization of the fan instability: Electron flux relaxation, Phys. Plasmas, 13(12), 122301, doi:10.1063/1.2372464.

Krafft, C., and A. S. Volokitin (2016), Electron acceleration by Langmuir waves produced by a decay cascade, Astrophys. J., 821(2), 99 , doi:10.3847/0004-637X/821/2/99.

Krafft, C., A. Volokitin, and A. Zaslavsky (2005), Saturation of the fan instability: Nonlinear merging of resonances, Phys. Plasmas, 12(11), 112309, doi:10.1063/1.2118727.

Krafft, C., A. Volokitin, and A. Zaslavsky (2010), Nonlinear dynamics of resonant interactions between wave packets and particle distributions with loss-cone-like structures, Phys. Rev. E, 82(6), 066402, doi:10.1103/PhysRevE.82.066402.

Krafft, C., A. S. Volokitin, and V. V. Krasnoselskikh (2013), Interaction of energetic particles with waves in strongly inhomogeneous solar wind plasmas, Astrophys. J., 778, $111-122$, doi:10.1088/0004-637X/778/2/111.

Krafft, C., A. S. Volokitin, V. V. Krasnoselskikh, and T. D. de Wit (2014), Waveforms of Langmuir turbulence in inhomogeneous solar wind plasmas, J. Geophys. Res. Space Physics, 119, 9369-9382, doi:10.1002/2014JA020329.

Krafft, C., A. S. Volokitin, and V. V. Krasnoselskikh (2015), Langmuir wave decay in inhomogeneous solar wind plasmas: Simulation results, Astrophys. J., 809, 176-193, doi:10.1088/0004-637X/809/2/176.

Krasnoselskikh, V. V., V. V. Lobzin, K. Musatenko, J. Soucek, J. S. Pickett, and I. H. Cairns (2007), Beam-plasma interaction in randomly inhomogeneous plasmas and statistical properties of small-amplitude Langmuir waves in the solar wind and electron foreshock, J. Geophys. Res., 112, A10109, doi:10.1029/2006JA012212.

LaBelle, J., I. H. Cairns, and C. A. Kletzing (2010), Electric field statistics and modulation characteristics of bursty Langmuir waves observed in the cusp, J. Geophys. Res., 115, A10317, doi:10.1029/2010JA015277.

Li, B., I. H. Cairns, P. A. Robinson, J. LaBelle, and C. A. Kletzing (2010), Waveform and envelope field statistics for waves with stochastically driven amplitudes, Phys. Plasmas, 17(3), 032110, doi:10.1063/1.3353092.

Neugebauer, M. (1975), The enhancement of solar wind fluctuations at the proton thermal gyroradius, J. Geophys. Res., 80, 998-1002, doi:10.1029/JA080i007p00998.

O'Neil, T. M., J. H. Winfrey, and J. H. Malmberg (1971), Nonlinear interaction of a small cold beam and a plasma, Phys. Fluids, 14, 1204-1212, doi:10.1063/1.1693587.

Pearson, K. (1895), Contributions to the mathematical theory of evolution. II. Skew variation in homogeneous material, Philos. Trans. R. Soc. London, Ser. A, 186, 343-414, doi:10.1098/rsta.1895.0010.

Píša, D., G. B. Hospodarsky, W. S. Kurth, O. Santolík, J. Souček, D. A. Gurnett, A. Masters, and M. E. Hill (2015), Statistics of Langmuir wave amplitudes observed inside Saturn's foreshock by the Cassini spacecraft, J. Geophys. Res. Space Physics, 120, 2531-2542, doi:10.1002/2014JA020560.

Podladchikova, O., B. Lefebvre, V. Krasnoselskikh, and V. Podladchikov (2003), Classification of probability densities on the basis of Pearson's curves with application to coronal heating simulations, Nonlinear Processes Geophys., 10, 323-333.

Press, W. H., S. A. Teukolsky, W. T. Vetterling, and B. P. Flannery (1992), Numerical Recipes in FORTRAN. The Art of Scientific Computing, Cambridge Univ. Press, New York.

Reid, H. A. S., and E. P Kontar (2017), Langmuir wave electric fields induced by electron beams in the heliosphere, Astron. Astrophys., 598, A44, doi:10.1051/0004-6361/201629697.

Robinson, P. A. (1992), Clumpy Langmuir waves in type III radio sources, Sol. Phys., 139, 147-163, doi:10.1007/BF00147886.

Robinson, P. A. (1995), Stochastic wave growth, Phys. Plasmas, 2, 1466-1479, doi:10.1063/1.871362.

Robinson, P. A., I. H. Cairns, and D. A. Gurnett (1993), Clumpy Langmuir waves in type III radio sources - Comparison of stochastic-growth theory with observations, Astrophys. J., 407, 790-800, doi:10.1086/172560.

Smith, D. F., and D. Sime (1979), Origin of plasma-wave clumping in type III solar radio burst sources, Astrophys. J., 233, 998-1004, doi:10.1086/157463.

Soucek, J., V. Krasnoselskikh, T. Dudok de Wit, J. Pickett, and C. Kletzing (2005), Nonlinear decay of foreshock Langmuir waves in the presence of plasma inhomogeneities: Theory and Cluster observations, J. Geophys. Res., 110, A08102, doi:10.1029/2004JA010977. 
Vedenov, A. A. (1967), Theory of a weakly turbulent plasma, Rev. Plasma Phys., 3, 229-276.

Vidojević, S., A. Zaslavsky, M. Maksimović, M. Dražić, and O. Atanacković (2011), Statistical analysis of Langmuir waves associated with type III radio bursts: I. Wind observations, Baltic Astron., 20, 596-599.

Volokitin, A., and C. Krafft (2004), Interaction of suprathermal electron fluxes with lower hybrid waves, Phys. Plasmas, 11, 3165-3176, doi:10.1063/1.1715100.

Volokitin, A., and C. Krafft (2012), Velocity diffusion in plasma waves excited by electron beams, Plasma Phys. Controlled Fusion, 11, 085002, doi:10.1088/0741-3335/54/8/085002.

Volokitin, A., and C. Krafft (2016), Diffusion of energetic electrons in turbulent plasmas of the solar wind, Astrophys. J., 833, 166, doi:10.3847/1538-4357/833/2/166.

Volokitin, A., V. Krasnoselskikh, C. Krafft, and E. Kuznetsov (2013), Modelling of the beam-plasma interaction in a strongly inhomogeneous plasma, 1539, 78-81, 10.1063/1.4810994

Voshchepynets, A., and V. Krasnoselskikh (2015), Probabilistic model of beam-plasma interaction in randomly inhomogeneous solar wind, J. Geophys. Res. Space Physics, 120, 10,139-10,158, doi:10.1002/2015JA021705.

Voshchepynets, A., V. Krasnoselskikh, A. Artemyev, and A. Volokitin (2015), Probabilistic model of beam-plasma interaction in randomly inhomogeneous plasma, Astrophys. J., 807, 38, doi:10.1088/0004-637X/807/1/38.

Zakharov, V. E. (1972), Collapse of Langmuir waves, Sov. J. Exp. Theor. Phys., 35, 908-914.

Zaslavsky, A., C. Krafft, and A. Volokitin (2006), Stochastic processes of particle trapping and detrapping by a wave in a magnetized plasma, Phys. Rev. E, 73(1), 016406, doi:10.1103/PhysRevE.73.016406.

Zaslavsky, A., C. Krafft, and A. Volokitin (2007), Loss-cone instability: Wave saturation by particle trapping, Phys. Plasmas, 14(12), 122302, doi:10.1063/1.2799621. 